\title{
Gradient trajectory analysis of a scalar field with external intermittency
}

\author{
JUAN PEDRO MELLADO†, LIPO \\ WANG AND NORBERT PETERS \\ Institut für Technische Verbrennung, RWTH Aachen University, Templergrabenst. 64, 52056 Aachen, \\ Germany
}

(Received 12 June 2008 and in revised form 5 January 2009)

The passive scalar field of a temporally evolving shear layer is investigated using gradient trajectories as a means to analyse the scalar probability density function and the conditional scalar dissipation rate in the presence of external intermittency. These results are of significance for turbulent combustion, where improved predictions of the statistics of the conditional dissipation rate are needed in several models. First, the variation of the conventional first and second moments of the conditional dissipation rate across the layer is quantitatively documented in detail. A strong dependence of the conditional dissipation rate on the lateral position and on the conditioning value of the scalar is observed. The dependence on the transverse distance to the centre-plane partially explains the double-hump profile usually reported when this dependence is ignored. The variation with the scalar observed in the ratio between the second and first moments would invalidate certain assumptions commonly done in turbulent combustion. It is also seen that conditioning on the scalar does not reduce the fluctuation of the dissipation rate with respect to unconditional values. Next, the role of external intermittency in these results is investigated. For that purpose, the flow is partitioned into different zones based on different types of gradient trajectories passing through each point, thereby introducing non-local information in comparison with the standard turbulent/non-turbulent separation based on the conventional intermittency function. In addition to the homogeneous outer regions, three zones are identified: a turbulent zone, a turbulence interface and quasi-laminar diffusion layers. The relative contribution from each of these zones to the conventional intermittency factor is reported. The statistics are then conditioned on each of these zones, and the spatial variation of the scalar distribution and of the conditional scalar dissipation rate is explained in terms of the observed zonal statistics. For the Reynolds numbers of the present simulation, between 1500 and 3000 based on the vorticity thickness and the velocity difference, and a Schmidt number equal to 1, it results that the major contribution to both statistics is due to the turbulence interfaces. At the same time, the turbulent zone shows a distinct behaviour, being approximately homogeneous but anisotropic.

\section{Introduction}

Free turbulent flows are encountered very often in engineering applications and geophysical flows, and the associated phenomena of turbulent entrainment and 
external intermittency are key features of turbulence, not only from the fundamental point of view but also from a practical one (Dimotakis 2005; Hunt, Eames \& Westerweel 2006). In spite of their long-standing recognition, a better understanding of these phenomena is still needed, specially if existing turbulent models are to be improved; an important example is the conditional scalar dissipation rate inside the intermittent zone, a key quantity in turbulent combustion.

Free turbulent flows are characterized by having turbulent regions adjacent to non-turbulent ones, the latter being associated with a negligible value of vorticity fluctuation (Townsend 1976). This leads to an alternating local behaviour when turbulent structures erode the non-turbulent zone and non-vortical flow is entrained into the turbulent region, giving rise to the aforementioned concept of external intermittency and which is quantified by the intermittency factor. First results about this quantity date back to the work of Townsend (1948) on the cylinder wake, and measurements in different experimental configurations have been reported in the literature (Antonia 1981). The interface between the non-turbulent and turbulent regions was first studied by Corrsin \& Kistler (1955), who introduced the concept of a laminar superlayer and postulated that its thickness scales with the Kolmogorov length scale. This thin diffusive layer, considered as a surface, has been further characterized by different authors. In terms of experimental data, a comprehensive presentation can be found in LaRue \& Libby (1976), while theoretical work has been reviewed by Sreenivasan, Ramshankar \& Meneveau (1989).

Based on the partition of the flow into turbulent and non-turbulent parts, Kovasznay, Kibens \& Blackwelder (1970) introduced the conditioning of the statistics on the region of the flow in which they were taken, splitting the conventional statistics of the turbulent boundary layer into zonal statistics. As a consequence, part of the variation of the conventional statistics can be explained in terms of the variation of the intermittency factor but not all of it. They also plotted the conditional mean velocity and fluctuation intensities as functions of the distance to the instantaneous surface, showing a relatively smooth variation across the interface. The corresponding profile of the lateral derivative of the streamwise velocity fluctuation suggested a stronger variation of the vorticity fluctuation, but measurements were not accurate enough to show this expected behaviour clearly.

More detailed spatial analyses of this region have been carried out in the last years, paying attention to the variation of flow quantities across the interface (Hunt et al. 2006). This advance has been caused by an improvement in measurement techniques (Westerweel et al. 2002; Holzner et al. 2006) as well as by direct numerical simulations (DNS), the latter providing the complete fields inside the turbulent/nonturbulent transition region. Bisset, Hunt \& Rogers (2002), working with a wake behind a flat plate, continued the analysis of Kovasznay et al. (1970) by calculating flow properties as functions of the distance to the interface, with the advantage that the vorticity was now available from the simulation; the rapid increase of vorticity over a relatively small distance, as postulated by Corrsin \& Kistler (1955), is now clearly observed. Bisset et al. (2002) also remarked that one must make the distinction between the turbulent/non-turbulent interface and the laminar superlayer. The former is characterized by a continuous transition between the outer laminar stream and the turbulent core, with fluctuations inside, and contains the latter, much thinner. Their results show that the thickness of the whole interface is comparable to the Taylor microscale instead of the Kolmogorov length scale, but no definitive conclusion about these scalings is possible with that moderate Reynolds number of the order of 2000 based on the centre-plane mean velocity defect and the half-width 
of the wake. Mathew \& Basu (2002) simulate a temporally evolving round wake, and their results suggest that small-scale entrainment (nibbling) might be comparable to that induced by the large-scale motion (engulfment) or even prevalent. Holzner et al. $(2007,2008)$ considered the details of the small-scale process of entrainment by which a fluid particle, initially in the outer laminar region, acquires vorticity as it enters the turbulent zone, comparing DNS with laboratory measurement in shear-free turbulence generated by an oscillating grid, in a Eulerian (Holzner et al. 2007) as well as in a Lagrangian (Holzner et al. 2008) context. They show that vorticity is first increased by viscous effects until the enstrophy production term dominates the enstrophy generation, the viscous term becoming then negative.

The idea that the turbulence interface might represent more than just a thin diffusive layer and that it might have an effect on the statistics of the whole flow was already hypothesized by Effelsberg \& Peters (1983), who considered this transition zone between the non-turbulent and the turbulent regions as a separate layer, with a particular structure different from that inside the fully turbulent zone and the free stream. With a simplified picture of this layer, they formulated a composite probability density function (p.d.f.) for the passive scalar, relating the four parameters of the model to the first four moments of the scalar field. Results using the plane turbulent wake data from LaRue \& Libby (1976) show the separation of the scalar p.d.f. into contributions from the different regions. However, the scalar profile assumed inside the transition region lacks a physical foundation apart from being a monotonous decrease towards the outer stream, and consequently a more detailed analysis of this zone is still needed.

This paper continues this study of the turbulent/non-turbulent transition region by means of a DNS of a non-reacting temporally evolving shear layer. More specifically, a partition of the flow into well distinguishable zones using gradient trajectories is applied, discussing then zonal statistics related to a passive scalar $Z$ in each of the resulting regions. Emphasis is made on the scalar p.d.f. $P_{Z}\left(Z^{\prime}\right)$, the scalar dissipation p.d.f. $P_{\chi}\left(\chi^{\prime}\right)$ and the statistical dependence between $\chi$ and $Z$, in particular the average scalar dissipation rate conditioned on the scalar $\bar{\chi}_{Z}\left(Z^{\prime}\right)$ and the corresponding second moment $\sigma_{\chi \mid Z}\left(Z^{\prime}\right)$. The local scalar dissipation rate is defined by $\chi=2 D|\nabla Z|^{2}, D$ being the scalar diffusivity, and the apostrophe denotes the sample-space variable of the corresponding random variable. The variations with the lateral distance to the centre-plane of the shear layer and with time are investigated in detail, comparing conventional with zonal statistics. Some statistics conditioned on the interface are clearly dependent on the position of this interface, as occurs with the mean velocities and whose variation is precisely used by Bisset et al. (2002) to explain the large-scale motion. In many other analysis these variations are ignored, and averages are taken along the complete interface. It will be shown below that the conditional scalar dissipation rate also experiences significant changes along the interface in the shear layer.

The present work focuses on the passive scalar and its dissipation rate because of its relevance to non-premixed turbulent combustion (Libby \& Williams 1994; Bilger 2000; Peters 2000). Conditional moment closure and p.d.f. methods require explicitly the mean dissipation rate (or the filtered values in the context of largeeddy simulations) conditioned on the different scalars, and the flamelet model needs the statistical characterization of the variable $\chi$ near the stoichiometric mixture fraction isosurface. However, the accuracy of several models commonly used is still insufficient in many circumstances (cf. Mellado, Friedrich \& Sarkar 2007 and the references therein). The analysis presented in this work is especially significant within 
the flamelet context, since the flamelet transformation is defined in terms of the gradient trajectories of the mixture fraction $Z$. Regions of stoichiometric mixtures, where the flame is approximately located, are often within the turbulent/non-turbulent interface, and the flamelet transformation has to be performed there.

Information about the relation between the scalar and the conditional scalar dissipation rate is therefore of primary importance. The current understanding is that the scalar distribution is Gaussian if and only if the dissipation rate and the scalar itself are independent, a result that has been shown by theory, measurements and simulations for the case of homogeneous turbulence (cf. Warhaft 2000; Mi 2006 and the references therein). Mi (2006) suggests that the same behaviour should appear in general, in particular in free turbulent flows, and this proposition is supported by the experimental data from turbulent jets reported by several authors in the past (Anselmet, Djeridi \& Furlachier 1994; Mi \& Antonia 1995; Tong \& Warhaft 1995). These data also show how the scalar dependence of the conditional mean dissipation rate varies as the measurement location is moved across the mixing region. On the other hand, Chen \& Mansour (1997) and Starner et al. (1997) showed a more or less constant conditional mean across the hydrogen jet diffusion flame, suggesting that reacting flows may differ from non-reacting flows in this matter. Related measurements have been presented by Bilger (1993) and Markides \& Mastorakos (2006), but their configurations do not have external intermittency. Detailed analysis about this lateral variation based on DNS of free turbulent flows has not been reported yet, to the authors' knowledge. Mell et al. (1994) suggested that no significant variation is appreciated, but they considered a scalar mixing layer inside an isotropic turbulent flow without mean shear as done in Bilger (1993). Pantano, Sarkar \& Williams (2003) studied heat release effects on the profiles of $\bar{\chi}_{Z}$ in a shear layer, but they used volume averaging to improve statistical convergence. This work tries to provide part of this missing detailed DNS analysis; the results presented here agree with the experimental data from non-reacting flows listed before in that the conditional scalar dissipation rate varies in space, and this lateral variation of $\bar{\chi}_{Z}\left(Z^{\prime}\right)$ is related to the external intermittency and the turbulence interface.

However, the main new contribution to the topic consists in the application of gradient trajectory analysis to the study of the scalar field in the presence of external intermittency. Gradient trajectories were already used by Peters \& Trouillet (2002) to study the central region of a shear layer and have successfully been employed in the analysis of homogeneous shear turbulence (Wang \& Peters 2006; Peters \& Wang 2007). This technique allows the definition of the so-called dissipation elements as regions of space from which the same minimum and maximum points are reached by gradient trajectories. Conditioning on these points provides better understanding of the scaling of some significant two-point correlations, like the scalar difference. The mean linear distance between minimum and maximum points is of the order of the Taylor scale, indicating that there exist anisotropic regions much larger than the Kolmogorov or the Batchelor scales which are diffusively connected. In free turbulent flows we have to cope, in addition, with trajectories outside of the turbulent region, coming from or heading towards the outer irrotational stream, and precisely this particularity can be used to identify the turbulent/non-turbulent interfaces.

The paper is organized as follows: $\S 2$ describes the relevant aspects of the simulation and the numerical algorithm employed. Section 3 presents the statistics to be investigated in the paper, namely the scalar p.d.f. and the scalar dissipation rate conditioned on the scalar. Section 4 describes the gradient trajectory approach and the partition resulting from it. Next, $\S 5$ repeats the analysis of the previous conventional 
statistics but now conditioned on each of the zones. The paper concludes with a discussion relating the zonal statistics with the original conventional statistics and summarizing the implications of the results for turbulent combustion models.

\section{Description of the numerical simulation}

The conservation equations of mass, momentum and energy, along with the transport equation of a passive scalar, $Z$, are solved with a numerical algorithm based on that reported by Pantano \& Sarkar (2002) and Stanley, Sarkar \& Mellado (2002), with a few modifications to improve the resolution. The convective term is written in the skew-symmetric form proposed by Erlebacher et al. (1990) in order to reduce aliasing errors (Kravchenko \& Moin 1997). The diffusion terms are treated in explicit form. The spatial first- and second-order derivatives are computed using a sixth-order compact Padé scheme (Lele 1992). It is one-sided at the non-periodic boundaries (top and bottom), having globally fourth-order accuracy (Carpenter, Gottlieb \& Abarbanel 1993). The advancement in time is performed with a low-storage fourthorder Runge-Kutta scheme (Williamson 1980). The boundary conditions in the nonperiodic directions are implemented in characteristic non-reflective form (Thompson 1990).

The streamwise direction is $O x$; the crosswise direction is $O y$; and the coordinate $z$ moves in the spanwise direction. Flow variables are statistically homogeneous in planes $x O z$, and these are used to compute the different statistics, which depend then on the crosswise coordinate $y$ and the time $t$. The origin in the $O y$ direction is chosen at the centre-plane. The passive scalar, $Z$, has the homogeneous value 0 in the upper stream and 1 in the lower stream. The governing equations, written in Cartesian coordinates, are discretized on a structured uniform mesh of size $2048 \times 512 \times 1024$ with equal grid spacing in every direction. Long streamwise and spanwise domain sizes are employed to improve statistical convergence, a known difficulty of conditional statistics (Antonia 1981).

The convective Mach number is set equal to 0.3 so that compressibility effects are negligible (Pantano \& Sarkar 2002). The ratio $\eta / \Delta x$, where $\eta$ is the Kolmogorov scale $\eta=\left(v^{3} / \bar{\epsilon}\right)^{1 / 4}$, with $\bar{\epsilon}$ the mean turbulent dissipation rate calculated at the centreplane and $v$ the kinematic viscosity, is about 1 in the interval of time in which the flow is analysed. Note that this value is more than twice the resolution used in previous related works (Pantano \& Sarkar 2002; Pantano et al. 2003), but this level of resolution is needed for the gradient trajectory analysis, as discussed later. Consequently, the Reynolds number is smaller than usual for this simulation size, attaining a maximum value at the end of the simulation of $\operatorname{Re}_{\omega}=\delta_{\omega} \Delta U / \nu \simeq 3000$, based on the vorticity thickness $\delta_{\omega}$ and the velocity difference across the shear layer $\Delta U$. The corresponding Taylor-scale Reynolds number is $R e_{\lambda}=u^{\prime} \lambda_{x} / v \simeq 85$, where $u^{\prime}$ is the longitudinal velocity root mean square (r.m.s.), and the Taylor microscale is $\lambda_{x}=u^{\prime} /\left[\overline{\left(\partial u^{\prime} / \partial x\right)^{2}}\right]^{1 / 2}$. The Prandtl number is 0.7 ; the ratio of heat capacities is 1.4; and the Schmidt number is 1.0 .

The initial condition consists of a hyperbolic-tangent mean profile with vorticity thickness $\delta_{\omega, 0}$ for the streamwise velocity and a broadband random Gaussian velocity field as perturbation, with a spectrum proportional to $\kappa^{2} \exp \left(-2 \kappa / \kappa_{0}\right), \kappa$ being the wavenumber, that peaks at the wavelength $4 \delta_{\omega, 0}$ and with a turbulence intensity of $q / \Delta U=0.3$, where $q^{2}=u^{\prime 2}+v^{\prime 2}+w^{\prime 2}$. An initial condition with half the peak wavelength and a turbulence intensity equal to 0.1 , as indicated in Pantano \& Sarkar (2002), was also considered, but the Reynolds number in this case is too small, 


$\begin{array}{ccccccccc} & R e_{\omega} & R e_{t} & R e_{\lambda} & \delta_{\omega} / \delta_{\omega, 0} & \delta_{\omega} / \lambda_{x} & \delta_{\omega} / \eta & u^{\prime} / \Delta U & q / \Delta U \\ \tau_{1} & 1615 & 397 & 74 & 11.1 & 4.0 & 73.9 & 0.19 & 0.29 \\ \tau_{2} & 3018 & 585 & 85 & 20.7 & 6.4 & 122.6 & 0.18 & 0.29\end{array}$

TABLE 1. Reynolds numbers $R e_{\omega}=\Delta U \delta_{\omega} / v, R e_{t}=\left(q^{2} / 2\right)^{2} /(\bar{\epsilon} v)$ and $R e_{\lambda}=u^{\prime} \lambda_{x} / \nu$, length scale ratios and turbulence intensities at times $\tau_{1}=t_{1} \Delta U / \delta_{\omega, 0}=92.9$ and $\tau_{2}=183.0$. Values at the centre-plane are used for the mean turbulent dissipation rate $\bar{\epsilon}$, the Kolmogorov length scale $\eta$, the Taylor microscale $\lambda_{x}$ and the turbulence intensities.

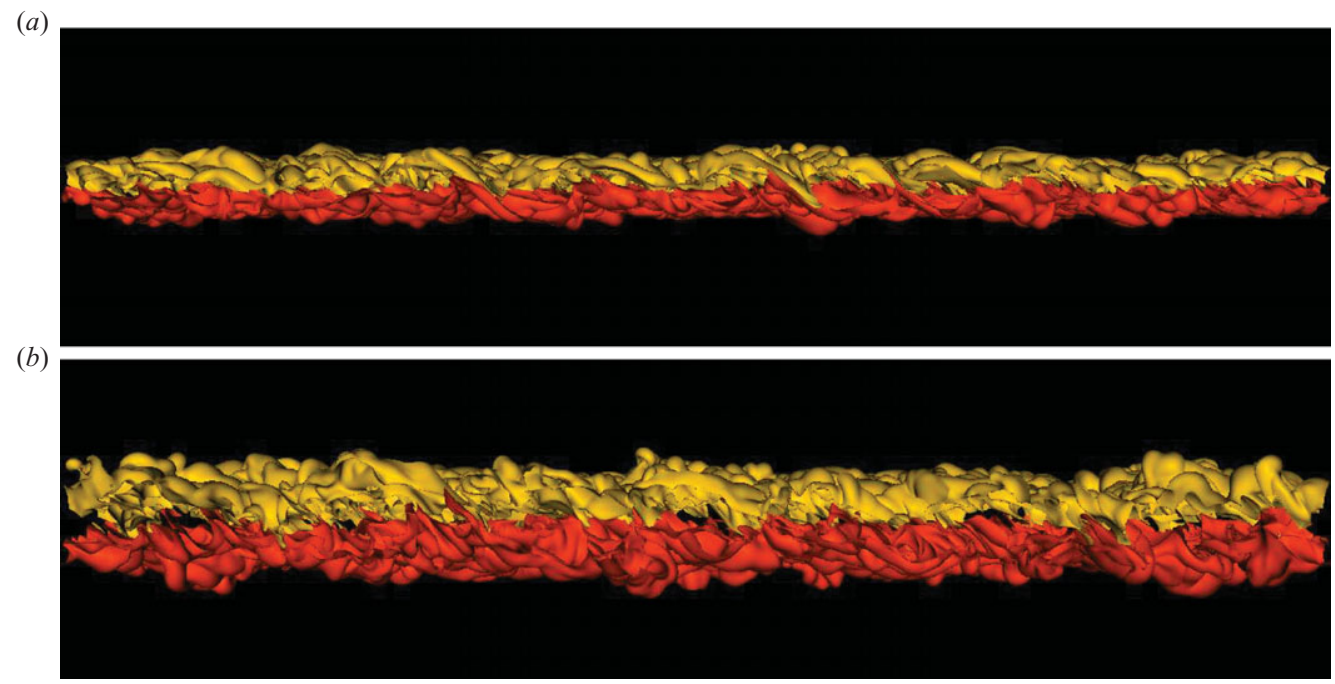

FIGURE 1. Lateral view of scalar isosurfaces $Z=0.05$ (yellow) and $Z=0.95$ (red) at $(a)$ $\tau_{1}=92.9\left(\delta_{\omega} / \delta_{\omega, 0}=11.1\right)$ and $(b) \tau_{2}=183.0\left(\delta_{\omega} / \delta_{\omega, 0}=20.7\right)$. Upper stream moves leftwards; lower flows rightwards.

and this initial perturbation led to a longer initial transient before the approximate self-similar behaviour was achieved.

The turbulent flow is now briefly characterized, some of its parameters being summarized in table 1 . The initial vorticity thickness is used to define the nondimensional time as

$$
\tau=t \Delta U / \delta_{\omega, 0}
$$

because it is this length, more than the momentum thickness, which best represents the spatial lateral variation of the statistics to be discussed in this paper. Four scalar fields are considered in the analysis presented in this work at equidistant times between $\tau_{1}=92.9$ and $\tau_{2}=183.0$. A visualization of the flow at these two times is presented in figure 1 , where the passive scalar field, $Z$, is depicted through two isosurfaces at the edge of the mixing region. The corresponding vorticity thickness at these times are $\delta_{\omega} / \delta_{\omega, 0}=11.1$ and 20.7 , so that the thickness of the mixing layer has increased over 10 times the initial one, and the four fields span a thickness variation of a factor of 2 . At the final time $\delta_{\omega}$ is about $25 \%$ of the vertical domain extent, and the simulation is stopped because of the influence from the top and bottom boundaries for a thicker layer. 
(a)

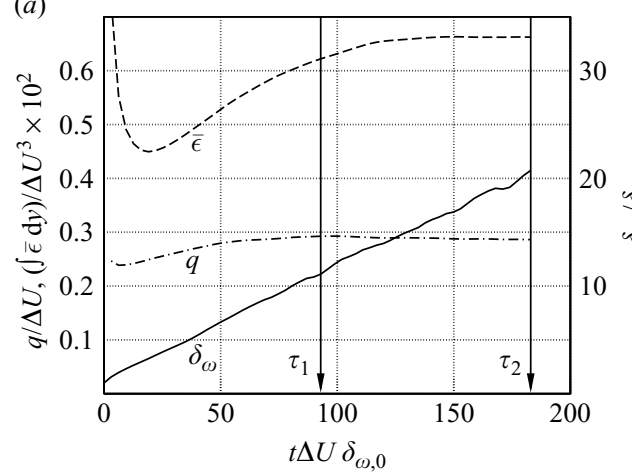

(b)

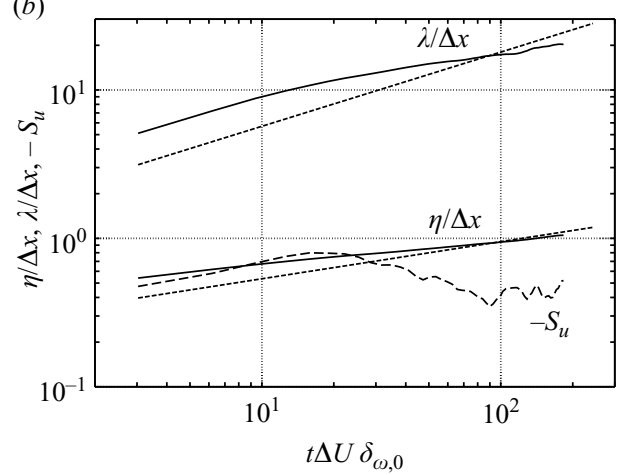

FIGURE 2. Temporal evolution of different statistics characterizing the flow, as indicated. Vertical lines indicate the time interval between $\tau_{1}=92.9$ and $\tau_{2}=183.0$ considered in the study.

The temporal evolution of the vorticity thickness is shown in figure $2(a)$ and the expected linear growth is clearly observed. After the initial transient, the corresponding momentum thickness growth rate approaches $0.018 \Delta U$ at the end of the simulation, a value comparable to 0.016 reported by Rogers \& Moser (1994) and to 0.018 reported by Pantano \& Sarkar (2002), within the interval 0.014-0.022 quoted in the first reference. The integral across the layer of $\bar{\epsilon}$, only a function of time, is included in that figure. According to inviscid scaling, this integral quantity should approach a constant value, since the velocity scale $\Delta U$ imposed on this flow is constant itself. A tendency towards this state is observed during the last half of the simulation, with a variation of about $6 \%$ over the time interval used in this study, whereas the vorticity thickness approximately doubles in the same period of time. The turbulence intensity $q / \Delta U$ at the centre-plane is also plotted, and it approaches 0.29 , which agrees with the value 0.26 calculated from the results of Rogers \& Moser (1994) and Pantano \& Sarkar (2002).

Figure 2(b) shows the evolution of the Kolmogorov length scale $\eta$ and the Taylor microscale $\lambda_{x}$, comparing them with the expected self-similar scalings $\tau^{1 / 4}$ and $\tau^{1 / 2}$, respectively. These curves show that the flow is close to, though still developing towards, the self-similar behaviour of the shear layer. Last, the skewness of the longitudinal derivative of the streamwise velocity is included in the figure, showing a value oscillating about -0.44 , which agrees with the data from other turbulent flows (Sreenivasan \& Antonia 1997).

\section{Conventional statistics}

In terms of one-point statistics, all the information regarding the random variables $Z$ and $\chi$ is contained in the joint distribution $P_{\chi Z}\left(\chi^{\prime}, Z^{\prime} ; y, t\right)$, which varies across the layer and in time. It is clear that in terms of two-point statistics inside the diffusive range these two quantities are related. Unfortunately, when the sample is constructed only with data of individual planes $x \mathrm{Oz}$, this sample is too small and the statistical convergence too poor for those joint p.d.f.s. For that reason, only part of that information is reported, namely the first two moments of the conditional p.d.f. $P_{\chi \mid Z}\left(\chi^{\prime} \mid Z=Z^{\prime} ; y, t\right)$ and the marginal distributions $P_{Z}\left(Z^{\prime} ; y, t\right)$ and $P_{\chi}\left(\chi^{\prime} ; y, t\right)$. The first conditional moment is the conditional mean dissipation rate $\bar{\chi}_{Z}\left(Z^{\prime} ; y, t\right)$, already introduced before, and the second conditional moment will be denoted by 

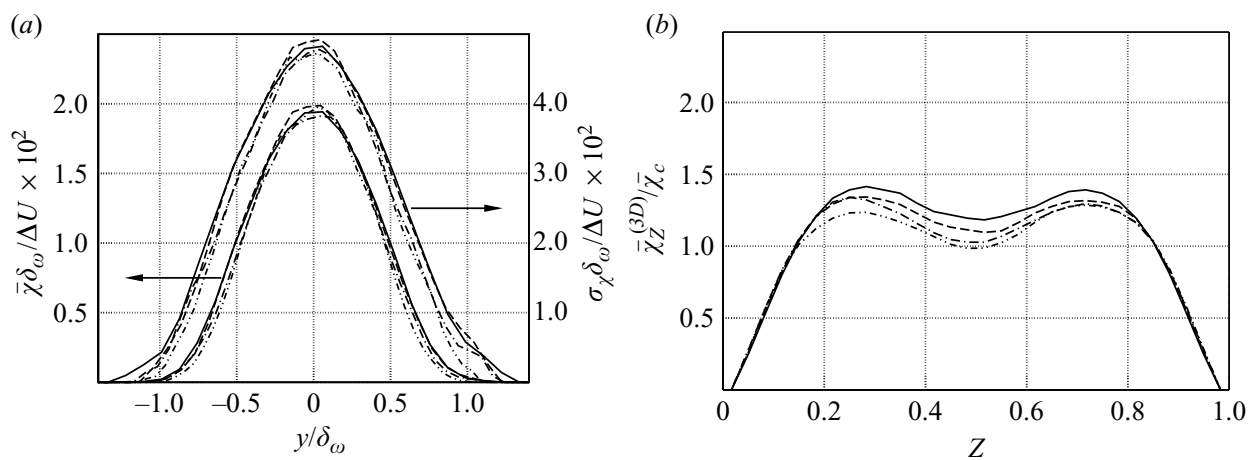

FIGURE 3. Profiles of the unconditional scalar dissipation rate $(a)$ plotted using self-similar variables: solid, $\tau_{1}=92.9$; dashed, $\tau=122.8$; dot-dashed, $\tau=152.8$; dot-dot-dashed, $\tau_{2}=183.0$. Average conditional scalar dissipation rate $(b)$ calculated using the whole field at those same times.

$$
\begin{aligned}
& \sigma_{\chi \mid Z}\left(Z^{\prime} ; y, t\right): \\
& \quad\left[\sigma_{\chi \mid Z}\left(Z^{\prime} ; y, t\right)\right]^{2}=\int_{0}^{\infty} \chi^{\prime 2} P_{\chi \mid Z}\left(\chi^{\prime} \mid Z=Z^{\prime} ; y, t\right) \mathrm{d} \chi^{\prime}-\left[\bar{\chi}_{Z}\left(Z^{\prime} ; y, t\right)\right]^{2} .
\end{aligned}
$$

Besides, the conditional mean $\bar{\chi}_{Z}$ is related to the unconditional average $\bar{\chi}(y, t)$ through the p.d.f. of the scalar according to

$$
\bar{\chi}(y, t)=\int_{0}^{1} \bar{\chi}_{Z}\left(Z^{\prime} ; y, t\right) P_{Z}\left(Z^{\prime} ; y, t\right) \mathrm{d} Z^{\prime},
$$

and a similar relation holds between the unconditional variance $\left[\sigma_{\chi}(y, t)\right]^{2}$ and the conditional one $\left(\sigma_{\chi \mid Z}\right)^{2}$; it is therefore also interesting to consider both $\bar{\chi}$ and $\sigma_{\chi}$.

This section describes these statistics without consideration of the field partition introduced later for deeper analysis, and the name conventional statistics is employed as in Kovasznay et al. (1970) to distinguish them from the zonal statistics discussed in $\S 5$. Some of the results shown in this section about $\bar{\chi}$ and $P_{Z}$ are extensively documented in the literature, but it is convenient to briefly introduce them here for completeness and for later reference. One final note in terms of notation: the distinction between random variable and sample-space variable is dropped hereafter.

Profiles of $\bar{\chi}(y, t)$ are plotted in figure 3(a) in terms of the self-similar variable

$$
\xi=y / \delta_{\omega}(t)
$$

and normalized by $\Delta U / \delta_{\omega}(t)$. The approximate collapse of the curves at different times is again a manifestation of the self-similar behaviour of this type of flow, which for the case of $\bar{\chi}$ already appears at this moderate Reynolds number. Curves of the standard deviation $\sigma_{\chi}(y, t)$ are also included in the same plot, showing values slightly larger than twice the mean dissipation and a broader profile. The order of magnitude of $\sigma_{\chi} / \bar{\chi}$ agrees with that reported in Effelsberg \& Peters (1988), who measured ratios between 1 and 2 in a turbulent jet, and that showed by Markides \& Mastorakos (2006), between 1 and 3. The centre-plane dissipation rate $\bar{\chi}_{c}(t)$ will be used as a reference throughout the paper to normalize the conditional values at each time. Figure $3(b)$ shows the conditional mean scalar dissipation rate calculated using the complete field, where the two-hump profile agrees with the results of previous 
(a)

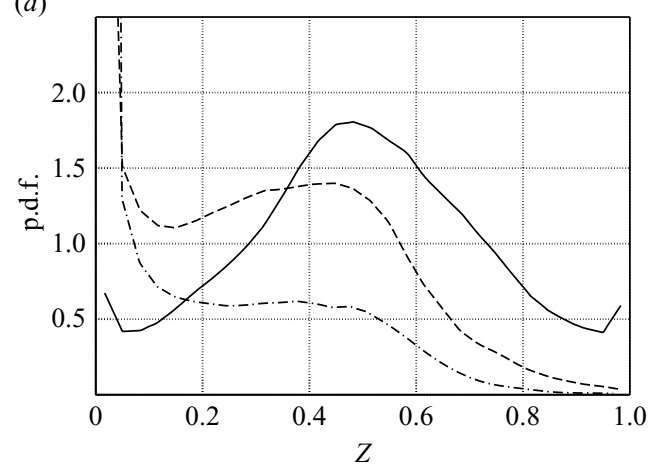

(b)

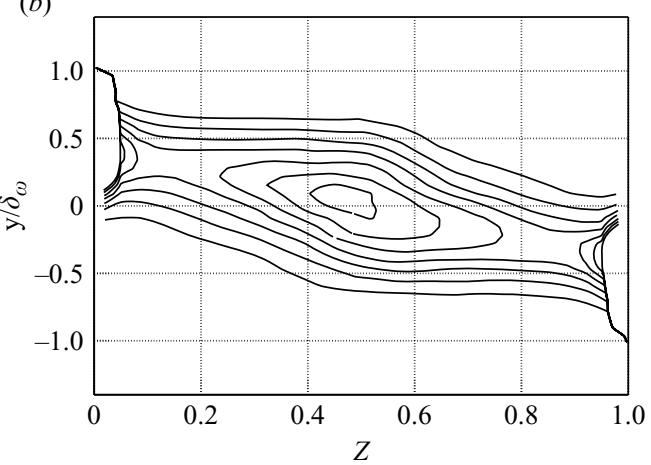

FiguRE 4. Scalar p.d.f. at $\tau_{2}=183.0$. Distributions $(a)$ at different heights: solid, $y=0$; dashed, $y=\delta_{\omega} / 4$; dot-dashed, $y=\delta_{\omega} / 2$. Contours $(b)$ of the function $P_{Z}(Z, \xi)$ every 0.25 , starting at 0.25 .

investigations in shear layers without heat release (Peters \& Trouillet 2002; Pantano et al. 2003).

Next, the curve $P_{Z}(Z ; y, t)$ as a function of $Z$ is shown for different values of the self-similar variable, $\xi$, in figure $4(a)$ at the final time $\tau_{2}=183.0\left(\delta_{\omega} / \delta_{\omega, 0}=20.7\right)$. The character of the p.d.f. is predominantly non-marching, the local maximum of the distribution staying at $Z=0.5$ for different crosswise positions and decreasing as we move towards the outer streams. This behaviour has been documented in the literature as a signature of dominant spanwise rollers and a relatively strong external intermittency (Rogers \& Moser 1994; Cortesi et al. 2001; Picket \& Ghandhi 2002). An alternative representation of the spatial variation with the crosswise coordinate is provided by the isocontours in figure 4(b), as used in Picket \& Ghandhi (2002) to improve quantitative comparison. This figure shows the contour levels of the function $P_{Z}(Z, \xi)$, the scalar $Z$ in abscissas and the normalized crosswise coordinate $\xi$ in ordinates. Both types of representation, profiles and contour plots, will be used extensively throughout the paper. The combination of a diagonal band representative of a certain degree of marching behaviour and the concentration of probability around the centre on the figure, representative of the non-marching behaviour, can be observed, and it is in agreement with the simulations presented in Cortesi et al. (2001) using random initial perturbations of the mean shear profile, as we have here. The symmetry $P_{Z}(Z ; y, t)=P_{Z}(1-Z ;-y, t)$ is also seen.

The time evolution of the scalar p.d.f. is described by comparing figure 5 , at time $\tau_{1}=92.9$, with the final time already shown in figure 4 . It is observed that the general hybrid structure does not change and that the use of the self-similar variable $\xi$ approximately collapses the two plots, and there is only a mild broadening in scalar space of the central peak which could be understood as a small tendency towards a more marching character. Note that the shear layer thickness has doubled from the first time to the last one, and so has the Reynolds number $R e_{\omega}$, but this is still moderate, and a mixing transition in the structure of the p.d.f. (Dimotakis 2000) might appear at later times.

The third statistic is the mean scalar dissipation rate conditioned on the scalar, $\bar{\chi}_{Z}(Z ; y, t)$, and it is shown in figure 6 . Profiles as functions of the scalar, $Z$, at different distances $y$ from the centre-plane are shown, as indicated. A strong dependence on this distance is observed. The structure of this dependence with the 


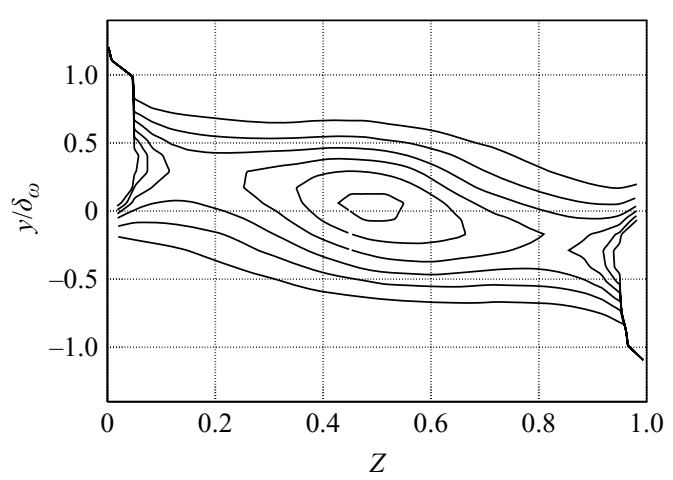

FIgURE 5. Scalar p.d.f. at $\tau_{1}=92.9$. Contours of the function $P_{Z}(Z ; \xi)$ every 0.25 , starting at 0.25 .
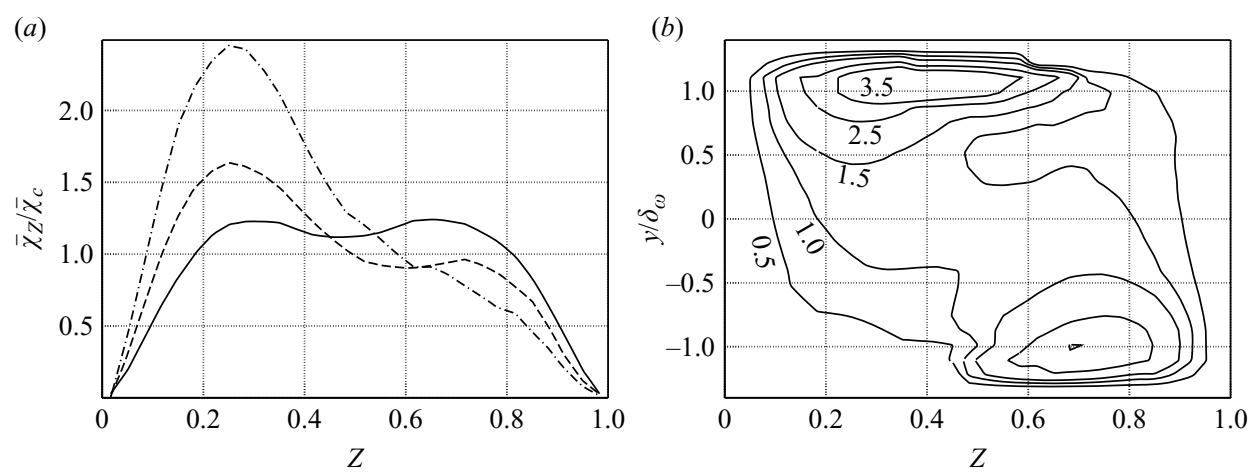

FIGURE 6. Conditional mean scalar dissipation rate between $\tau_{1}=92.9$ and $\tau_{2}=183.0$. Profiles (a) at different heights: solid, $y=0$; dashed, $y=\delta_{\omega} / 2$; dot-dashed, $y=3 \delta_{\omega} / 4$. Contours $(b)$ of the function $\bar{\chi}_{Z}(Z, \xi) / \bar{\chi}_{c}(t)$.

crosswise coordinate is more clearly seen in figure $6(b)$, where isocontours of $\bar{\chi}_{z}$ as a function of $Z$ and $\xi$ are presented. Results show that the conditional mean scalar dissipation rate is more or less flat in the centre, dropping of course to zero at the limits $Z=0$ and $Z=1$, but, as we move away from the middle plane, it develops two clear peaks centred around a scalar value that is biased towards the corresponding outer laminar one. One of these peaks is located somewhere between $Z=0.2$ and $Z=0.4$ towards the upper free stream and the second one around the corresponding symmetric value towards the lower stream. This lateral variation of $\bar{\chi}_{Z}$ supports the experimental results about non-reacting flows found in the literature (Anselmet et al. 1994; Mi \& Antonia 1995; Tong \& Warhaft 1995). The variation of $\bar{\chi}_{Z} / \bar{\chi}_{c}$ with $Z$ is a clear indication that the random variables $Z$ and $\chi$ are not independent.

This dependence on the lateral coordinate, in particular the strong values of dissipation at the edge of the mixing layer, is not observed in the unconditional profiles in figure $3(a)$ because the scalar p.d.f. drops to zero in those regions, as observed in figure 4 , approximately beyond $\xi=0.5$ and $\xi=-0.5$, and the combination of both through (3.2) yields the smooth decay of $\bar{\chi}$ from the centre maximum value towards zero in the outer regions. The average conditional dissipation shown in figure 6 is scaled with $\bar{\chi}_{c}(t)$, the corresponding unconditional value at the centre-plane. The 
(a)

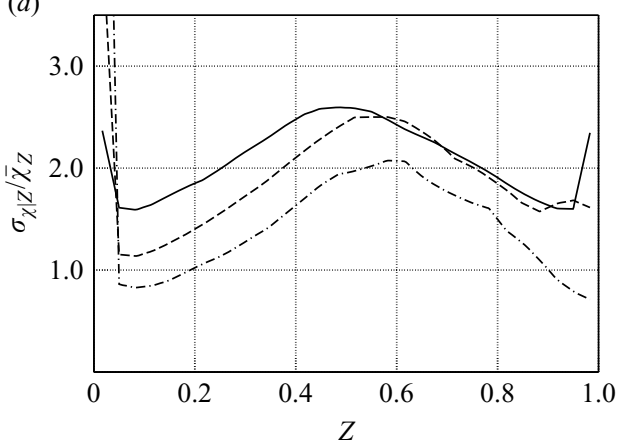

(b)

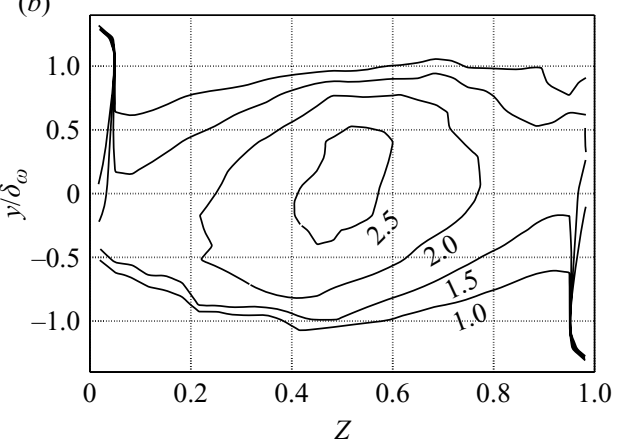

FIGURE 7. Ratio between conditional standard deviation $\sigma_{\chi \mid Z}$ and conditional mean between $\tau_{1}=92.9$ and $\tau_{2}=183.0$. Profiles $(a)$ at different heights: solid, $y=0$; dashed, $y=\delta_{\omega} / 2$; dot-dashed, $y=3 \delta_{\omega} / 4$. Contours $(b)$ of the function $\left(\sigma_{\chi \mid Z} / \bar{\chi}_{Z}\right)(Z, \xi)$.

central plateau of $\bar{\chi}_{Z}$ lays then around 1 and is consistent with the fact that this plateau contributes the most to the unconditional profile.

The conditional second moment $\sigma_{\chi \mid Z}(Z ; y, t)$ is presented in figure 7 , showing $(a)$ individual profiles as well as $(b)$ the contour plot. This second-order moment will be discussed as a ratio with respect to the first moment, i.e. $\sigma_{\chi \mid Z} / \bar{\chi}_{Z}$, as normally done in the literature. This ratio is greater than 1 over most of the mixing region, a result that has been observed in the laboratory (Effelsberg \& Peters 1988; Markides \& Mastorakos 2006), attaining values higher than 2 in the centre, which agrees with data from homogeneous shear turbulence (Peters \& Wang 2007). It is also noted that these conditional values are comparable to the unconditional values shown in figure 3 and indicate that the conditioning of $\chi$ on $Z$ does not reduce significantly the fluctuation of the dissipation rate in this configuration with respect to the unconditional one.

The variation of $\sigma_{\chi \mid Z} / \bar{\chi}_{Z}$ occurs in physical space as well as in scalar space and is of relevance for turbulent combustion. The dependence on the lateral position $y$, along with that observed in the conditional mean in figure 6, implies that the post-processing of the whole volume in order to get details about the conditional distribution (for instance the conditional distribution $P_{\chi \mid Z}$ at stoichiometric surface needed in some combustion models) can lead to errors in the prediction of the statistical behaviour of the scalar dissipation rate. On the other hand, the dependence on $Z$ of the ratio $\sigma_{\chi \mid Z} / \bar{\chi}_{Z}$ is of significance for the possible functional relation between $\chi$ and $Z$, as now discussed. A relation of the form $\chi=\chi_{0} f(Z)$, where $f$ is some deterministic function and $\chi_{0}$ and $Z$ are statistically independent, is sometimes assumed in order to close the flamelet equations (Cook, Riley \& Kosály 1997; Pitsch, Chen \& Peters 1998). If this assumption is true, it is straightforward to show that the ratio $\sigma_{\chi \mid Z} / \bar{\chi}_{Z}$ should be independent of $Z$, but figure 7 clearly shows that that is not the case. Therefore, the assumption $\chi=\chi_{0} f(Z), \chi_{0}$ and $Z$ being independent, does not hold, and a more complicated relation between $\chi$ and $Z$ exists in this shear flow configuration.

The temporal evolution is mainly a broadening of the mixing region, and when the vorticity thickness is used to scale the crosswise coordinate the structure of the functions $\bar{\chi}_{Z}\left(Z ; y / \delta_{\omega}, t\right)$ and $\sigma_{\chi \mid Z}\left(Z ; y / \delta_{\omega}, t\right)$ does not vary significantly with time, at least within the statistical convergence and time interval achieved in this simulation. This result is used to average the self-similar functions related to the conditional scalar dissipation over the four fields between $\tau_{1}=92.9$ and $\tau_{2}=183.0$, and present smoother curves, as those in figures 6 and 7 . Hence, the vorticity thickness characterizes the 
lateral variation inside the shear layer, and the use of the self-similar variable defined by (3.3) captures the leading-order dependence on time. Corrections to this main dependence might appear as the Reynolds numbers increase beyond the moderate values achieved in this simulation, between $R e_{\omega}=1600$ and $R e_{\omega}=3000$.

The p.d.f. of the scalar dissipation rate was calculated as well, but external intermittency contributes to low values of the dissipation rate because of the outer quasi-homogeneous field, and that avoids any definitive conclusion about the possible skewness of the distribution ( $\mathrm{Su} \&$ Clemens 2003). The analysis of this p.d.f. is therefore deferred to $\S 5$, where the statistics are conditioned on the different flow zones. A conditioning on a particular scalar value would remove this problem, but, as already mentioned above, the observed variation across the layer of the conditional dissipation prevents us from making this choice.

A final note concerning the conditional mean dissipation is the following: the variation of $\bar{\chi}_{Z}$ with $y$ disappears if the whole three-dimensional flow is used to compute the conditional scalar dissipation, obtaining a curve with a hump on each side as shown in figure $3(b)$. Figure 6 shows that the cause of these two humps can be partly explained by the spatial structure of $\bar{\chi}_{Z}(Z ; y, t)$, in particular the two peaks that appear as one moves towards the outer irrotational stream, precisely in the ranges of scalar $0.2-0.4$ and $0.6-0.8$. However, there is also a (milder) two-hump profile at the centre-plane, as observed in figure 6 , and that is still to be understood. More important is to note that figure $3(b)$ underpredicts the maximum values of conditional mean dissipation, occurring at the upper and lower regions of the mixing region and shown in figure 6 , by a factor of 3 .

It is concluded from the results presented in this section that the relation between the scalar dissipation rate and the scalar itself varies across the shear layer. For our particular case, a turbulent shear flow, it seems plausible to think of a relation of this variation to the external intermittency, which increases as we move away from the centre-plane, and in particular that the peak values observed in the conditional mean may be formed in the turbulent/non-turbulent interface. This is investigated in the following sections.

\section{Space partition based on gradient trajectories}

External intermittency is commonly quantified by only one parameter, the intermittency factor $\gamma(y, t)$, based on the partition of the flow into two states, either non-turbulent if the vorticity magnitude is below a chosen threshold or turbulent in any other case (Townsend 1976); a review on the topic can be found in Antonia (1981). A passive scalar is sometimes used as a surrogate, using then a threshold in that scalar to distinguish between the two states (LaRue \& Libby 1976; Antonia 1981; Westerweel et al. 2002; Holzner et al. 2006) and thus introducing an alternative intermittency factor which in principle might differ from that based on the vorticity, specially for cases with Schmidt or Prandtl numbers very different from unity. This second approach is more related to our work because this paper focuses on the passive scalar, and the scalar gradient is used here to separate the outer homogeneous zones with negligible $\chi$ from the turbulent regions. Note however that our Schmidt number is 1 , and some of the previous laboratory experiments employ values about 2000 (Westerweel et al. 2002; Holzner et al. 2006), which could have an influence in some of the results. Figure 8 shows the scalar dissipation rate inside different vertical and horizontal planes at several times, in logarithmic scale and with a colour grade varying from black (lowest value) to white (highest value). The lamellar structure of 
(a)

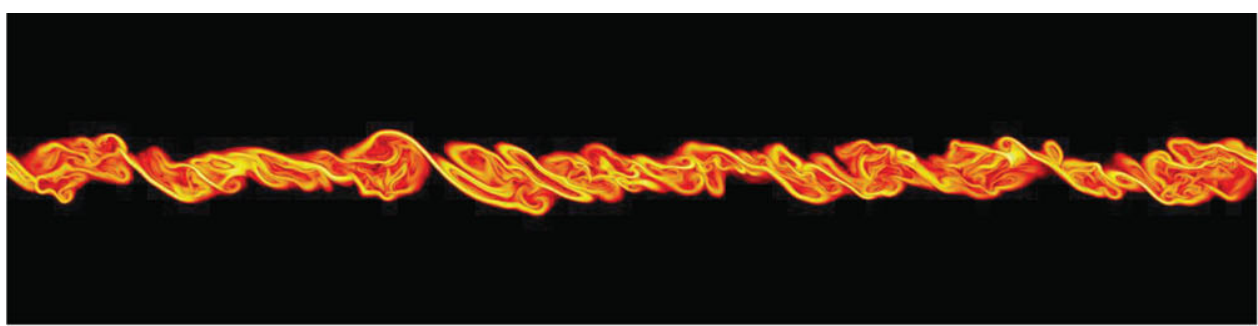

(b)

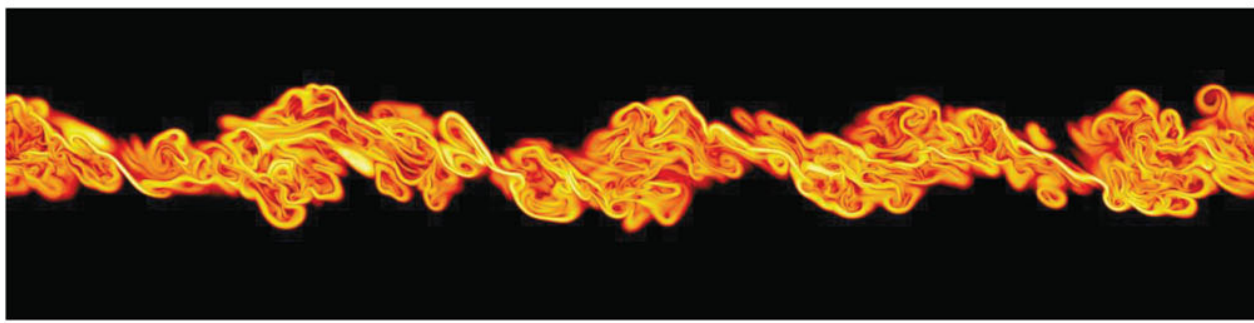

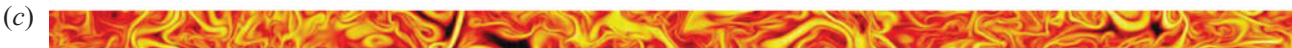

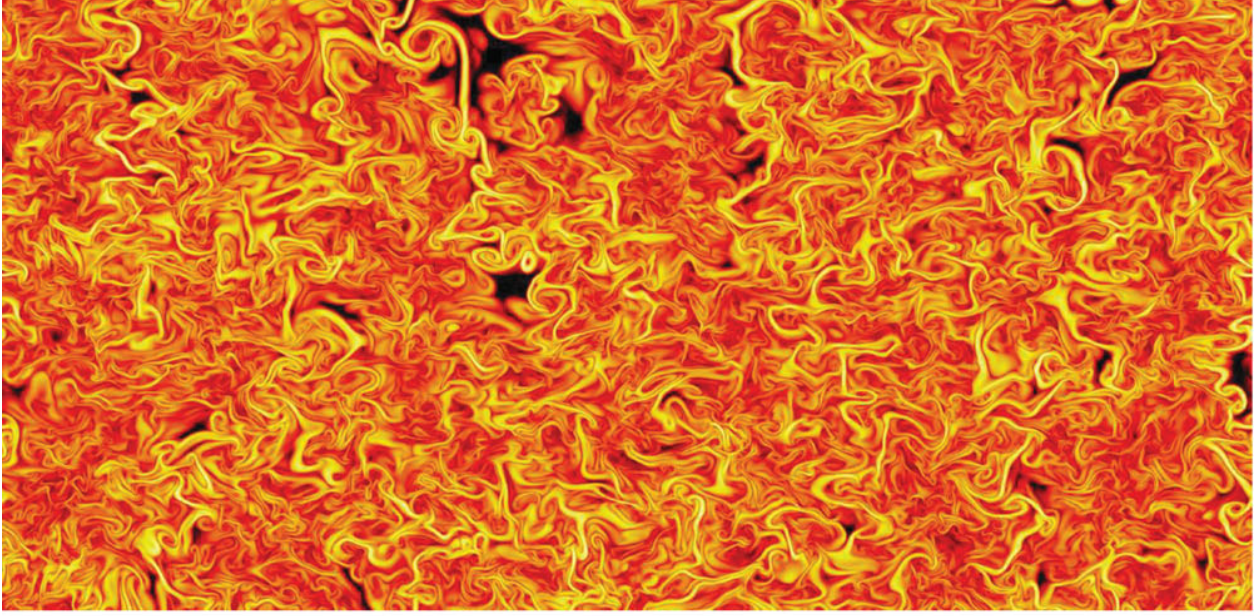

(d)

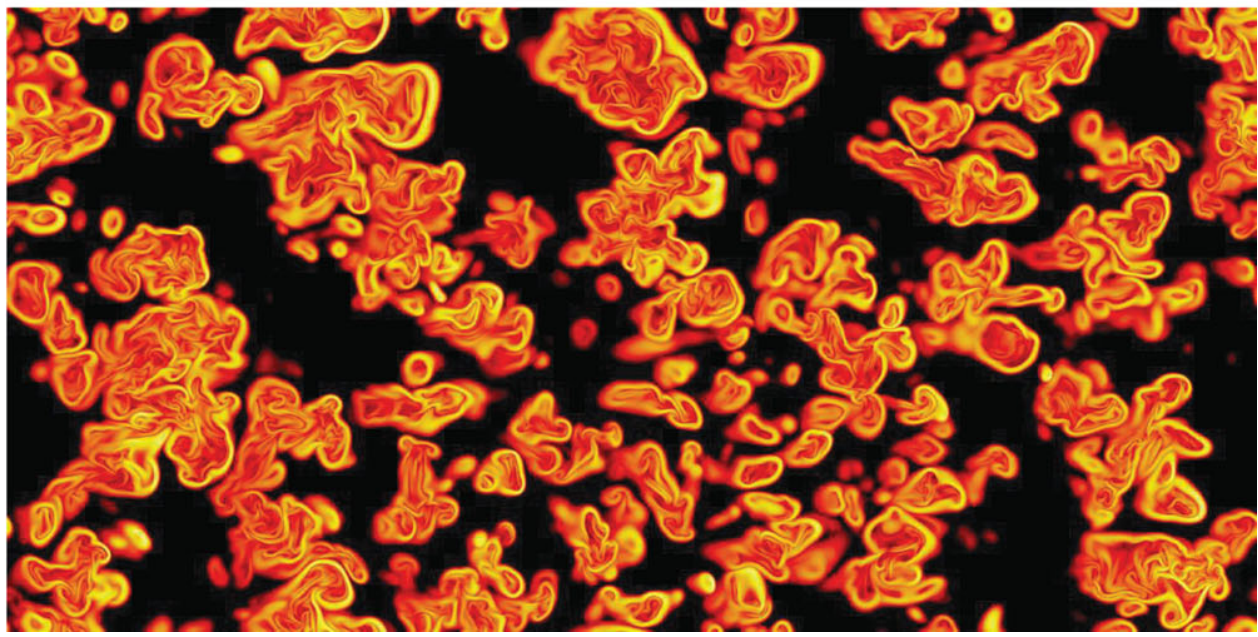

FiguRE 8. Logarithm of field $\chi$, colour scale starting at black for threshold $10^{-4} \bar{\chi}_{c}(t)$, increasing in the sequence red-yellow-white: (a) xOy plane at $\tau_{1}=92.9 ;(b) x O y$ plane at $\tau_{2}=183.0 ;(c) x O z$ at $y / \delta_{\omega}=0$ (centre-plane) at $\tau_{2} ;(d) x O z$ plane at $y / \delta_{\omega}=0.5$ at $\tau_{2}$. 
the field $\chi$ has been already shown by Su \& Clemens (2003) in turbulent plane jet measurements and by Kushnir, Schumacher \& Brandt (2006) in stationary isotropic turbulence DNS. A threshold is set in the plots of figure 8 at $10^{-4} \bar{\chi}_{c}(t)$, and external intermittency is then represented by the alternation of black free-stream and coloured turbulent zones. The ratio between the area occupied by the coloured zone and the total one in the horizontal planes, cases $(c)$ and $(d)$, are precisely the intermittency factors $\gamma\left(0, t_{2}\right)$ and $\gamma\left(0.5 \delta_{\omega}, t_{2}\right)$; the former has a large intermittency factor, close to 1 , but still pure fluid regions are observed to reach the centre-plane, whereas the latter has a value of $\gamma$ significantly smaller than 1 .

However, the turbulent/non-turbulent transition occurs across a region of finite size, as already described in the introduction. Effelsberg \& Peters (1983) considered the turbulent/non-turbulent transition zone as a third region and therefore introduced a second parameter describing the probability of this new zone. They emphasized that the characteristics of this transition zone might be relevant to the overall behaviour if it constitutes a relatively large fraction of the flow. One objective of this work is to show that gradient trajectories can be used in this context to actually identify this zone and characterize some of its properties. This transition zone will be referred to as the turbulent/non-turbulent interface or simply turbulence interface, following the recent literature and reserving the term laminar superlayer to the thin region dominated by diffusion effects inside this interface (Bisset et al. 2002; Westerweel, Fukushima \& Hunt 2005; Hunt et al. 2006; Holzner et al. 2008).

The study of a turbulent field based on gradient trajectories is presented in Wang \& Peters (2006), and details of the algorithm are also to be found there. The basic idea is that the gradient trajectory that passes through each point in space can be followed in the ascending direction until a maximum is detected and in the descending direction until a minimum is reached. Such points, at which the direction of the gradient trajectory is undefined, are the local extrema of the scalar field considered, $Z$ in our case, i.e. points at which the gradient of $Z$ vanishes and at which the Hessian are either positive-definite (minima) or negative-definite (maxima). A few modifications are needed for the present analysis because that original work considers a homogeneous turbulent flow without external intermittency. In the shear layer, we have to cope with the two free streams, which implies that some of the gradient trajectories head towards the outer irrotational stream without reaching an extremal point. In fact, these escaping trajectories are used to define the turbulence interface and to partition the flow. Thus, the general algorithm is as follows: The gradient trajectory at each point is calculated. If this gradient trajectory joins one minimum and one maximum, as represented by trajectories $\mathrm{A}$ in the sketch of figure 9, that point is said to be inside the turbulent zone, which is depicted by the two regions enclosed by the solid lines in that same figure. On the contrary, if the trajectory connects a maximum with the upper outer stream, where the scalar is $Z=0$, that point belongs to the upper turbulence interface. That case is represented by trajectories B in figure 9 . Correspondingly, if the trajectory moves from a minimum to the lower outer stream, where the scalar is $Z=1$, the point is inside the lower turbulence interface, trajectory $\mathrm{C}$ in figure 9. One last scenario is possible, namely that the trajectory connects the upper outer region with the lower one without an intermediate extremal point, as in case $\mathrm{D}$ in figure 9 . The points whose trajectories follow this behaviour define internal quasi-laminar diffusion layers.

The different zones just defined and sketched in figure 9 can be also qualitatively identified in the flow visualization presented in figure 8 , cases $(a)$ and $(b)$ showing the vertical planes. The turbulent zone embraces the core of the billows, where a random 


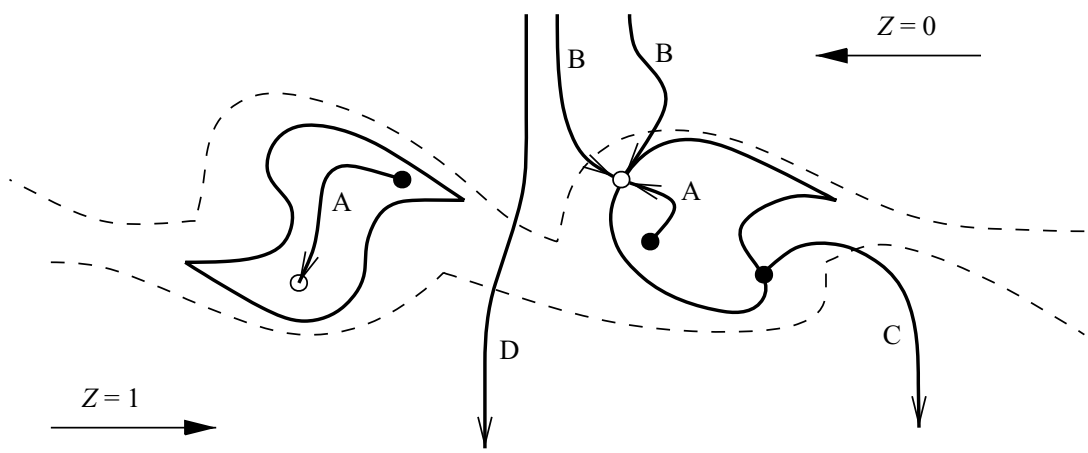

FIGURE 9. Flow partition based on gradient trajectories: A, trajectory from minimum to maximum, turbulent zone; $\mathrm{B}$, from upper stream to maximum, upper interface; $\mathrm{C}$, from minimum to lower stream, lower interface; $\mathrm{D}$, from upper stream to lower stream, quasi-laminar diffusion layers. Solid line separates turbulent zone from turbulence interface. Dashed line indicates threshold in the gradient magnitude to define the conventional intermittency function.

layered structure of the scalar gradient is observed. These billows are surrounded by the turbulence interfaces, a transition zone towards the outer non-turbulent streams (black). High values of the scalar gradient appear in the braid regions (white colour) and correspond to the quasi-laminar layers.

This identification of the turbulent zone is not based on any ad hoc threshold, and it will be shown in $\S 5$ that the turbulent zone so defined presents a very differentiated statistical behaviour. The corresponding interfaces are characterized by a monotonic variation of the scalar field from the outer homogeneous value to a maximum or minimum, respectively, but the gradient, and therefore the scalar dissipation, will fluctuate along the trajectory, and it is these fluctuations that we want to characterize. It is also noted that this partition is based on non-local information, in the sense that one point at a given distance from the centre-plane with a scalar value between the free-stream values might belong to the upper or the lower interface region, and the distinction is only possible by following the corresponding trajectory. In fact, it will be shown later that the upper interface often penetrates into the lower half of the layer and vice versa. This non-local approach allows to detect engulfed regions, as represented for instance by trajectory $\mathrm{C}$ in figure 9, which is not possible if the interface definition is based on a single-valued envelope surface.

However, an outer limit to the interfaces is also set $a d$ hoc by a threshold in the magnitude of the scalar gradient, below which the scalar is approximately a homogenous field. This second boundary, indicated by the thin dashed line in figure 9 , defines the conventional intermittency function and separates the non-turbulent zones from the turbulence interfaces. In figure 8, this boundary corresponds approximately to the contour defined by the black colour around the colour-coded mixing region. This differentiation between the outer non-turbulent zones and the turbulent/nonturbulent interface is introduced for several reasons. First, it is needed from the numerical point of view because the gradient approaches zero as one moves towards the outer homogeneous region, and below a threshold there is only noise, and the gradient direction is numerically undetermined. Second, this distinction is the conventional one used to define the intermittency factor and can be used to compare with traditional results using only this quantity. Finally, it is also useful to simplify possible models, since the p.d.f. of the scalar field in these non-turbulent regions is just a delta function at the corresponding outer value ( 0 or 1 in our case), and the 

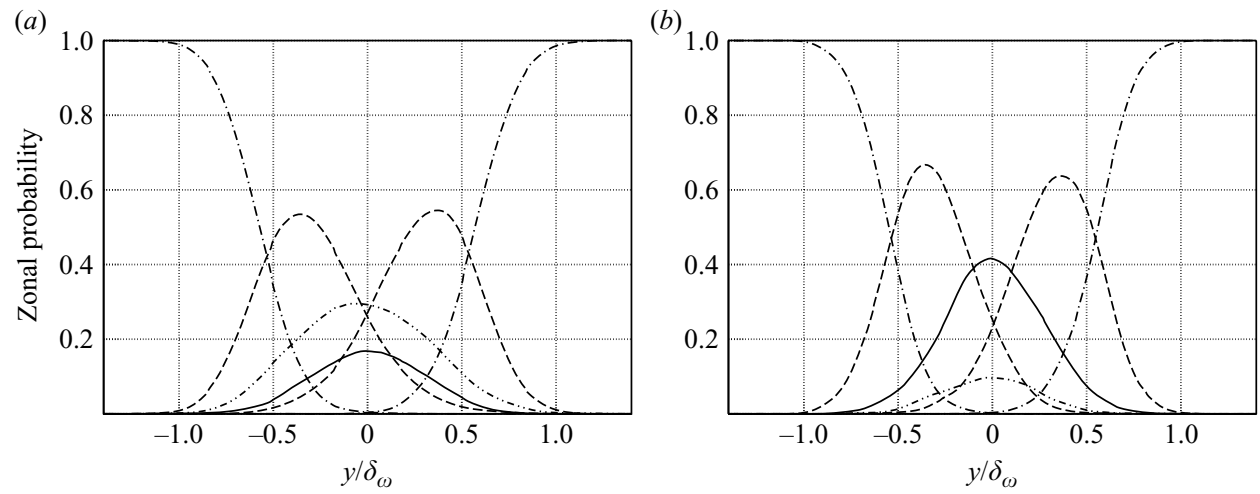

FIGURE 10. Profiles of zonal probability at $(a) \tau_{1}=92.9$ and $(b) \tau_{2}=183.0$ : solid, turbulent zone $\gamma_{t}(y, t)$; dashed, upper turbulence interface, $\gamma_{i}(y, t)$, and the lower one; dot-dashed, upper laminar zone, $\gamma_{n}(y, t)$, and the lower one; dot-dot-dashed, quasi-laminar diffusion layers, $\gamma_{q}(y, t)$.

scalar dissipation can be approximated by zero. Different approaches to define this separation can be found in the literature, and the choice made here is justified by the small sensitivity to the particular chosen threshold of the statistics considered in this work, as further discussed at the end of this section.

In summary, a point at a given distance $y$ from the centre-plane at a given time $t$ might belong to the upper or lower external non-turbulent zones, to the upper or lower interface, to the turbulent region or to the quasi-laminar diffusion layers. A probability of belonging to each of these zones can be calculated by the area fraction that each zone covers in each of the $x O z$ planes. These probabilities depend on the crosswise distance to the centre-plane and time, and they are plotted in figure 10 in terms of the self-similar variable $\xi$ defined by (3.3). The profile $\gamma_{n, u}(y, t)$ corresponds to the upper non-turbulent zone; the profile $\gamma_{i, u}(y, t)$ indicates the upper turbulence interface; the curve $\gamma_{t}(y, t)$ is the probability of the turbulent zone; and $\gamma_{q}(y, t)$ represents the quasi-laminar diffusion layers. The lower interface profile $\gamma_{i, l}(y, t)$ satisfies $\gamma_{i, l}(y, t)=\gamma_{i, u}(-y, t)$ from the symmetry of the configuration, as shown in the figure, and the same applies to the lower non-turbulent zone $\gamma_{n, l}(y, t)$; only one of these profiles is therefore considered hereafter to simplify the notation, and it is chosen $\gamma_{n} \equiv \gamma_{n, u}$ and $\gamma_{i} \equiv \gamma_{i, u}$. The conventional or total intermittency factor is

$$
\gamma(y, t)=\gamma_{t}(y, t)+\left[\gamma_{i}(y, t)+\gamma_{i}(-y, t)\right]+\gamma_{q}(y, t)=1-\left[\gamma_{n}(y, t)+\gamma_{n}(-y, t)\right] .
$$

The behaviour of the outer non-turbulent regions is as expected, dropping from 1 to a zero probability as we move deeper inside the mixing region. The turbulence interfaces peak at about $y= \pm 0.35 \delta_{\omega}(t)$ and drop asymmetrically to zero as the outer streams are approached. It is also observed that they penetrate deep into the opposite half of the mixing layer, as already mentioned before, and there is a finite non-negligible probability of finding the upper interface between $\xi=-0.5$ and the centre-plane and vice versa. The turbulent zone mainly concentrates between $-0.8 \delta_{\omega}(t)$ and $0.8 \delta_{\omega}(t)$.

Different times have qualitatively a similar distribution along $\xi$, as observed by comparing the two plots shown in figure 10, but the maximum of the profiles varies in time, specially noting that the contribution from the quasi-laminar layers decreases notoriously in favour of the turbulent regions. That temporal evolution is further 


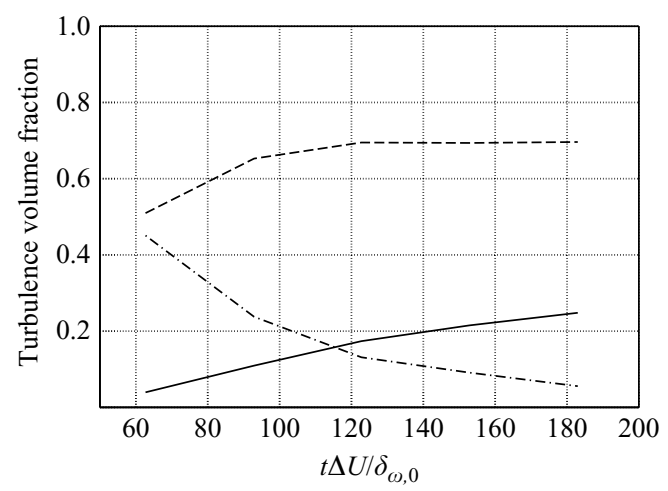

FigURE 11. Volume fraction of the complete turbulent region occupied by the different zones as a function of time: solid, turbulent zone; dashed, upper and lower interfaces added together; dot-dashed, quasi-laminar diffusion layers.

depicted in figure 11. The integrals along $\xi$ of the previous intermittency profiles have been calculated; these integrals indicate the volume occupied by each of the zones and the sum of the three contributions (turbulent core, interfaces and quasi-laminar layers) correspond to the integral of the total intermittency factor $\int \gamma(\xi, t) \mathrm{d} \xi$. The ratios of each of those first three integral quantities to the total intermittency represent the volume fraction in the whole turbulent mixing region of the turbulent zone, both turbulence interfaces and the quasi-laminar layers, respectively, and are plotted in figure 11. As already noticed, the relatively large contribution from the quasi-laminar layers decreases rapidly. It has already been said that the Reynolds number of the flow is moderate due to the resolution required by the algorithm to calculate the gradient trajectories, and that is probably the cause of the large values of $\gamma_{q}$ at early times. As the time evolves, i.e. as the Reynolds number increases, the volume fraction occupied by this type of flow structures decreases in favour of the turbulent ones. It is also observed that the turbulence interface occupies a portion of the flow significantly larger than the turbulent zone, and therefore the properties of these transition regions determine to a large extent the properties of the whole mixing layer, as suggested by Effelsberg \& Peters (1983), although it seems that the turbulent zone grows with respect to those interfaces, and it will be interesting to see the relative evolution of these two curves in figure 11 with an increasing Reynolds number.

This time evolution is also observed in the visualizations presented in figure $8(a, b)$. At an early time, the cores of the large-scale structures are relatively small, and there is a significant volume occupied by the braids, which can be identified with the quasi-laminar diffusion layers. As the flow develops, the billows increase in size, and so does the turbulent zone enclosed inside them relative to the braids, which at the same time seem to have more turbulent fluctuations inside them. Concomitantly, the volume occupied by the turbulence interface defining these billows also grows.

Independent of whatever behaviour is more probable at higher Reynolds numbers, the fact is that, for a moderate Reynolds number about 3000 as we have here, the global structure is dominated by turbulence interfaces, and at early times of the development of the shear layer the quasi-laminar diffusion zones are also of importance. This structure has a strong influence in the models to be developed.

Lastly, the effect of the threshold chosen to distinguish between the turbulence interface and the outer non-turbulent zone is now discussed. First, this threshold is 
varied to study the resolution requirements for the gradient trajectory analysis in the following way: If this external threshold is decreased, so that more space close to the outer homogeneous stream is considered inside the interface, the number of extrema detected by the algorithm should remain approximately the same because this new external region practically does not add new ones, its gradient trajectories going towards the extremal points existing in the turbulent zone. For the current simulation with a resolution of about $\eta / \Delta x \simeq 1.0$, a variation in the threshold value between $10^{-2} \bar{\chi}_{c}$ and $10^{-5} \bar{\chi}_{c}$ leads to a change of only $0.3 \%$ in the number of extremal points detected. However, preliminary analysis using smaller simulations with half that resolution led to a variation of about $50 \%$. This result indicates that $\eta / \Delta x \simeq 1$ for the numerical scheme described in $\S 2$ is appropriate, a resolution requirement for gradient trajectory analysis that is similar to that reported by Wang \& Peters (2006) for a homogeneous turbulent shear flow using a spectral scheme. Second, the influence of this threshold on the reported statistics needs to be considered. A nominal value $10^{-4} \bar{\chi}_{c}$ is used by default in all the results of this paper. When this value is increased by two orders of magnitude to $10^{-2} \bar{\chi}_{c}$, the integrated interface probability $\int \gamma_{i}(\xi, t) \mathrm{d} \xi$ is reduced by $15 \%$, with a corresponding increase of the volume fraction occupied by the non-turbulent zone. However, zonal statistics discussed in the following section remain globally the same, and therefore this threshold is irrelevant for the statistics presented in this work. Details of the plots in the limits $Z \rightarrow 0$ and $Z \rightarrow 1$ vary slightly, but we are not concerned with them in this paper.

\section{Zonal statistics}

The previous partition of the flow allows to write any distribution as

$$
\begin{aligned}
P(Z ; y, t)= & \gamma_{t}(y, t) P_{t}(Z ; y, t) \\
& +\gamma_{i, u}(y, t) P_{i, u}(Z ; y, t)+\gamma_{i, l}(y, t) P_{i, l}(Z ; y, t) \\
& +\gamma_{n, u}(y, t) P_{n, u}(Z ; y, t)+\gamma_{n, l}(y, t) P_{n, l}(Z ; y, t) \\
& +\gamma_{q}(y, t) P_{q}(Z ; y, t),
\end{aligned}
$$

where the subscript on the p.d.f.s on the right-hand side of the equation indicate that they are conditioned on the corresponding zone. The symmetries $\gamma_{i, l}(y, t)=\gamma_{i, u}(-y, t)$ and $\gamma_{n, l}(y, t)=\gamma_{n, u}(-y, t)$ can be used to simplify the previous expression, and in case of the scalar field we have in addition $P_{i, l}(Z ; y, t)=P_{i, u}(1-Z ;-y, t)$ and $P_{n, l}(Z ; y, t)=P_{n, u}(1-Z ;-y, t)$, which leads to

$$
\begin{aligned}
P_{Z}(Z ; y, t)= & \gamma_{t}(y, t) P_{Z, t}(Z ; y, t) \\
& +\gamma_{i}(y, t) P_{Z, i}(Z ; y, t)+\gamma_{i}(-y, t) P_{Z, i}(1-Z ;-y, t) \\
& +\gamma_{n}(y, t) P_{Z, n}(Z ; y, t)+\gamma_{n}(-y, t) P_{Z, n}(1-Z ;-y, t) \\
& +\gamma_{q}(y, t) P_{Z, q}(Z ; y, t),
\end{aligned}
$$

so that the distributions that need to be investigated are only three, namely $P_{t}, P_{i}$ and $P_{q}$, since that of the external non-turbulent zone is well approximated by a delta function. 
(a)

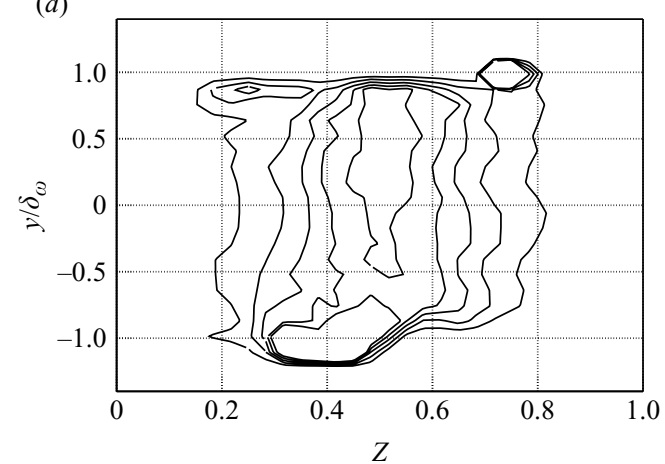

(b)

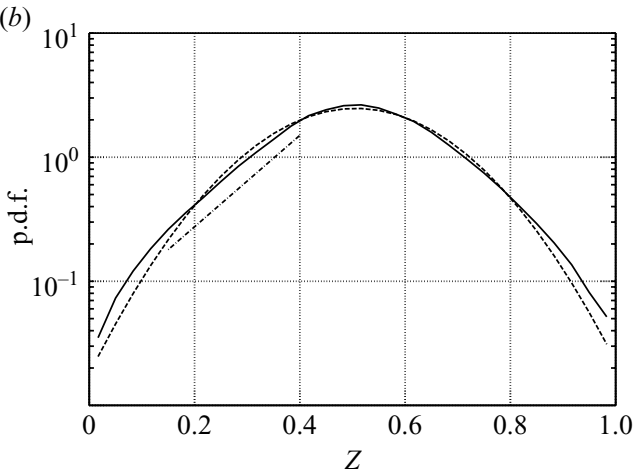

FIGURE 12. Scalar p.d.f. inside the turbulent zone at $\tau_{1}=92.9$. (a) Contours of the function $P_{Z, t}(Z, \xi)$ every 0.5, starting at 0.5. (b) Mean distribution including a reference Gaussian distribution (dashed) and exponential interval (dot-dashed).

Similarly, the p.d.f. of the scalar dissipation rate can be written as

$$
\begin{aligned}
P_{\chi}(\chi ; y, t)= & \gamma_{t}(y, t) P_{\chi, t}(\chi ; y, t) \\
& +\gamma_{i}(y, t) P_{\chi, i}(\chi ; y, t)+\gamma_{i}(-y, t) P_{\chi, i}(\chi ;-y, t) \\
& +\gamma_{n}(y, t) P_{\chi, n}(\chi ; y, t)+\gamma_{n}(-y, t) P_{\chi, n}(\chi ;-y, t) \\
& +\gamma_{q}(y, t) P_{\chi, q}(\chi ; y, t) .
\end{aligned}
$$

The same reasoning can be applied to the conditional moments, and the mean scalar dissipation rate conditioned on the scalar satisfies

$$
\begin{aligned}
\bar{\chi}_{Z}(Z ; y, t) P_{Z}(Z ; y, t)= & \gamma_{t}(y, t) \bar{\chi}_{Z, t}(Z ; y, t) P_{Z, t}(Z ; y, t) \\
& +\gamma_{i}(y, t) \bar{\chi}_{Z, i}(Z ; y, t) P_{Z, i}(Z ; y, t) \\
& +\gamma_{i}(-y, t) \bar{\chi}_{Z, i}(1-Z ;-y, t) P_{Z, i}(1-Z ;-y, t) \\
& +\gamma_{n}(y, t) \bar{\chi}_{Z, n}(Z ; y, t) P_{Z, n}(Z ; y, t) \\
& +\gamma_{n}(-y, t) \bar{\chi}_{Z, n}(1-Z ;-y, t) P_{Z, n}(1-Z ;-y, t) \\
& +\gamma_{q}(y, t) \bar{\chi}_{Z, q}(Z ; y, t) P_{Z, q}(Z ; y, t),
\end{aligned}
$$

once the existing symmetries have been used to reduce the number of statistics to be studied. Then, the additional quantities to be investigated are $\bar{\chi}_{Z, t}(Z ; y, t)$, $\bar{\chi}_{Z, i}(Z ; y, t)$ and $\bar{\chi}_{Z, q}(Z ; y, t)$, since the conditional mean scalar dissipation rate in the outer non-turbulent zone is close to zero. A similar partition can be written for the second-order quantity $\sigma_{\chi \mid Z}(Z ; y, t)$.

\subsection{Turbulent zone}

The scalar p.d.f. conditioned on the turbulent zone is shown in figure 12 at $\tau_{1}=92.9$. Figure 12(a) contains the contour lines of the function $P_{Z, t}(Z, \xi)$ and shows that the distribution is quite independent of the crosswise position, which indicates that the total scalar field inside the turbulent region, not only the fluctuation, is homogeneous to a good approximation, and the mean scalar gradient is almost zero. At the same time, this result allows to consider the whole turbulent zone to calculate the distribution, and the resulting density function $P_{Z, t}(Z)$ is presented in figure $12(b)$, where ordinates are plotted in logarithmic scale to magnify the tails. The distribution 
(a)

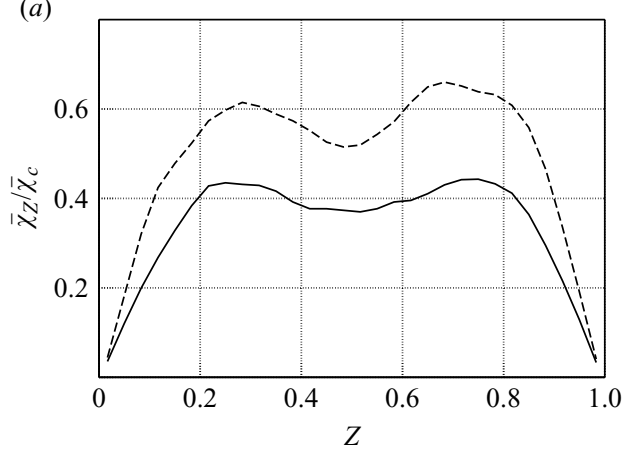

(b)

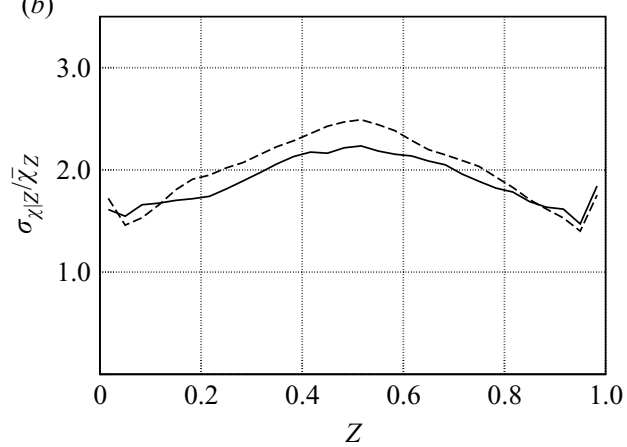

FIgURE 13. Conditional mean $(a)$ and standard deviation $(b)$ of the scalar dissipation rate inside the turbulent zone at different times: solid, $\tau_{1}=92.9$; dashed, $\tau_{2}=183.0$.

is approximately symmetric with respect to $Z=0.5$ with a standard deviation of 0.16 and a flatness of 3.04. Though these values suggest a Gaussian p.d.f., the comparison with it included in the figure shows that there are deviations (in addition to the fact that the field is bounded between 0 and 1). The beta p.d.f. was also calculated with the first two moments of the distribution, for comparison, and it showed even larger departures from the measurement. It seems more that two intervals with exponential behaviour are present.

Results in the literature normally relate the exponential tails in the scalar distribution to the presence of a mean gradient, provided that the Reynolds number is high enough and that the domain is large enough compared to the integral length scale (Warhaft 2000). However, Eswaran \& Pope (1988) also reports non-Gaussian behaviour in isotropic turbulence with a scalar field evolving from an initial bimodal distribution without a mean gradient. The results presented in figure $12(b)$ agree with the latter, and an interval of exponential trend emerges on both sides of the distribution before the external bounds of the passive scalar impose a faster decay towards zero near $Z=0$ and $Z=1$, although there is no strong mean gradient inside this turbulent zone. This behaviour probably originates in the genesis of the scalar fluctuations inside the turbulent region. The lamellar structure observed in the scalar gradient in figure 8 suggests that the scalar fluctuations are created when the steepgradient scalar sheets formed in the braid regions are engulfed into the turbulent zone by the main spanwise structures and the rib vortices. The turbulent state inside the turbulent zone corresponds then to an intermediate time in the development of a bimodal distribution towards the final Gaussian state shown in Eswaran \& Pope (1988). The difference with that work is that here the scalar fluctuation is continously maintained by the entrainment process, whereas there the scalar fluctuation decays.

These exponential tails in the distribution of the scalar imply that the conditional mean scalar dissipation rate cannot be constant (O'Brien \& Jiang 1991), which is precisely the behaviour of $\bar{\chi}_{z, t}$ seen in figure 13(a). This figure shows volume averages over the whole turbulent zone, which are reasonable because the contour plot (not presented) showed that the spatial variation along $y$ was again very small compared with the variation in scalar space. This $\mathrm{V}$ shape of $\bar{\chi}_{Z}$ relates the stronger dissipation events to the large departures of the mean value indicated by the exponential tails of $P_{Z}(Z)$ and has been already reported by Eswaran \& Pope (1988) in stationary isotropic turbulence DNS and by Jayesh \& Warhaft (1992) in grid turbulence measurements. The distinction between turbulent zone and turbulence interface based on extremal 


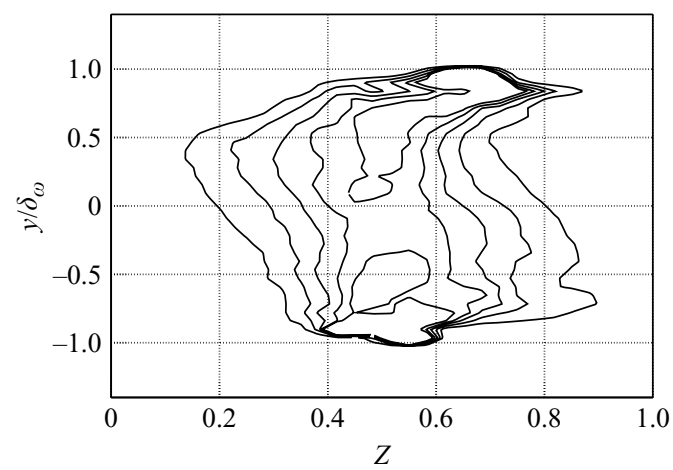

FIGURE 14. Scalar p.d.f. inside the turbulent zone at $\tau_{2}=183.0$. Contours of the function $P_{Z, t}(Z, \xi)$ every 0.5 , starting at 0.5 .

points, where $\chi=0$, forces eventually the fall of $\bar{\chi}_{Z}$ towards zero at the limits $Z \rightarrow 0$ and $Z \rightarrow 1$, and consequently two local maxima form at $Z \simeq 0.3$ and $Z \simeq 0.7$. This twohump shape is reminiscent of the conventional conditional mean scalar dissipation rate presented in figure 3(b); however, it cannot explain this figure because, first, the magnitude of $\bar{\chi}_{Z}$ inside the turbulent zone is small compared to the total conditional scalar dissipation of figure 6, and, second, the volume fraction occupied by the turbulent region is much smaller than that occupied by the turbulence interfaces.

The conditional standard deviation $\sigma_{\chi \mid Z}$ defined in (3.1) is also considered, and figure $13(b)$ shows the ratio $\sigma_{\chi \mid Z} / \bar{\chi}_{Z}$ calculated again using the complete turbulent zone, since the variation with $y$ was small. The magnitude is comparable to that of the unconditional quantities shown in figure 7 , showing as well a dependence with $Z$ that causes a variation of about $60 \%$ between $Z=0.5$ and the free-stream values. This ratio agrees with the results reported by Peters \& Wang (2007) for the case of homogeneous turbulence.

In terms of the time evolution of the previous quantities, the scalar dissipation remains approximately homogeneous across the mixing zone during the time interval accessible in this simulation, the values of the first and second moments varying between $\tau_{1}$ and $\tau_{2}$ as shown in figure 13. However, the scalar p.d.f. seems to initiate a change in structure, as observed by comparing times $\tau_{1}$ from figure 12 and time $\tau_{2}$ from figure 14, and the skewness starts to vary along $y$. It seems that a mean shear begins to develop inside the turbulent zone, but longer simulations are needed to assess this behaviour with the required detail.

The distribution of the dissipation rate $P_{\chi}(\chi)$ and associated parameters are plotted in figure 15. The previous results in this section suggest to compare this statistic with that of homogeneous turbulent flows. In this respect, the distribution of $\ln \chi$ shown in figure $15(a)$ is in very good agreement to that presented by Wang, Chen \& Brasseur (1999) and Vedula, Yeung \& Fox (2001) in stationary and freely decaying isotropic turbulence; the distribution of $\chi$ inside the turbulent zone is therefore approximately lognormal, with a slight negative skewness, as already indicated by Eswaran \& Pope (1988). The standard deviation, skewness and flatness are shown in figure $15(b)$, with characteristic values $\sigma_{\ln \chi} \simeq 1.6, S_{\ln \chi} \simeq-0.25$ and $F_{\ln \chi} \simeq 3.2$, but a small variation in time is observed. These values are similar to those reported in the previously indicated references. The fourth curve shown in figure $15(b)$ is the mean dissipation rate inside the turbulent zone normalized by the unconditional centre-plane value, a ratio that 

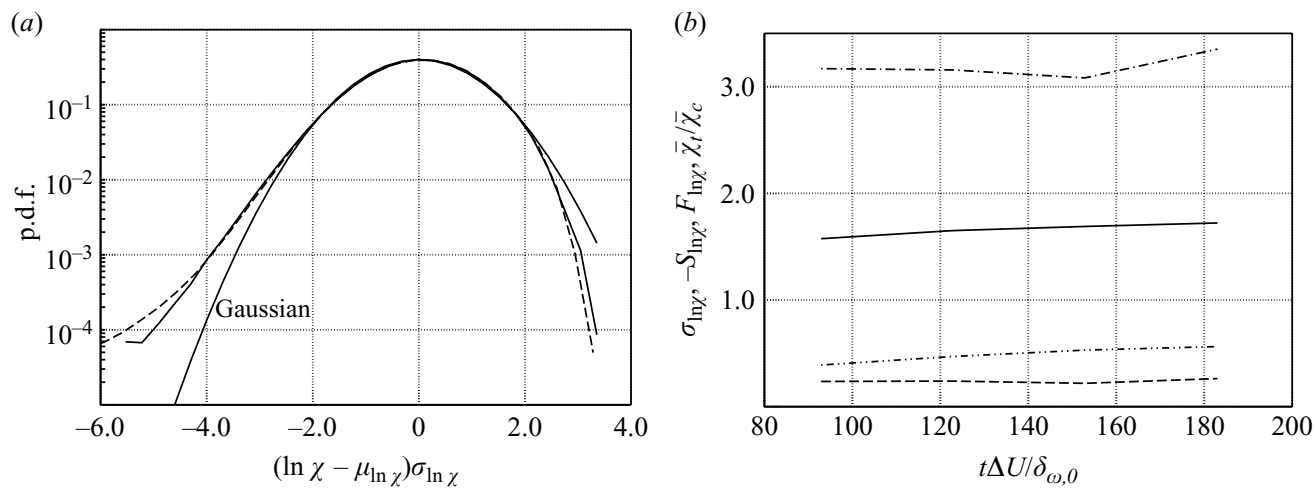

FIGURE 15. Probability distribution of the normalized scalar dissipation rate $(a)$ inside the turbulent zone at different times: solid, $\tau_{1}=92.9$; dashed, $\tau_{2}=183.0$. Time evolution $(b)$ of different parameters: solid, standard deviation $\sigma_{\ln \chi}$; dashed, skewness $S_{\ln \chi}$; dot-dashed, flatness $F_{\ln \chi} ;$ dot-dot-dashed, normalized mean dissipation rate $\bar{\chi}_{t} / \bar{\chi}_{c}$.
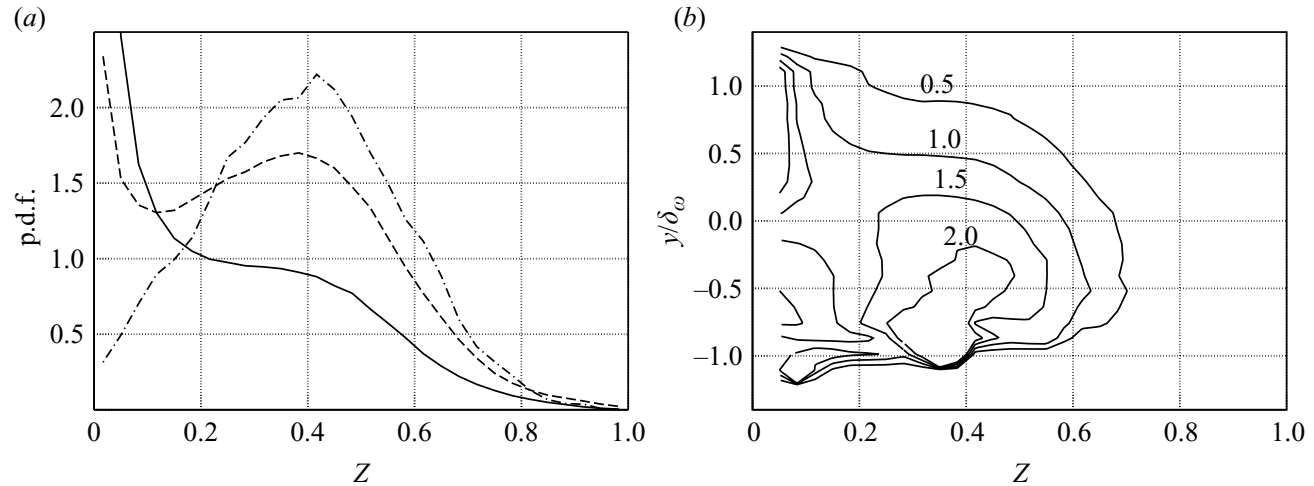

FIGURE 16. Scalar p.d.f. inside the upper turbulence interface at $\tau_{1}=92.9$. Distributions $(a)$ at different heights: solid, $y=\delta_{\omega} / 2$; dashed, $y=0$; dot-dashed, $y=-\delta_{\omega} / 2$. Contours $(b)$ of the function $P_{Z, i}(Z, \xi)$.

varies between 0.40 at $\tau_{1}$ and 0.56 at $\tau_{2}$ and is consistent with the conditional values already presented in figure 13 .

Deeper analysis of this turbulent zone, though relevant, falls out of the scope of this work. However, it was interesting to verify if isotropy conditions hold in addition to the observed homogeneity. For that purpose the statistics of the three components of the scalar gradient vector were computed separately. The results confirm that the flow is not isotropic inside this turbulent zone, since the mean of the spanwise component is zero, and the other two are positive with a ratio of the crosswise and the streamwise components that varies between 2 at $\tau_{1}$ and 1 at $\tau_{2}$.

\subsection{Turbulent/non-turbulent interface}

Statistics calculated inside the upper turbulence interface are now discussed. The lower interface obeys the symmetries with respect to the upper one described in $\S 4$, and it is used to double the sample size and improve statistical convergence.

The scalar distribution at the time $\tau_{1}=92.9$ is shown in figure 16 and a strong variation in the crosswise coordinate is observed. Far from the centre of the shear layer, beyond $\xi=0.5$, the distribution shows the strong peak at $Z=0$, the free-stream 


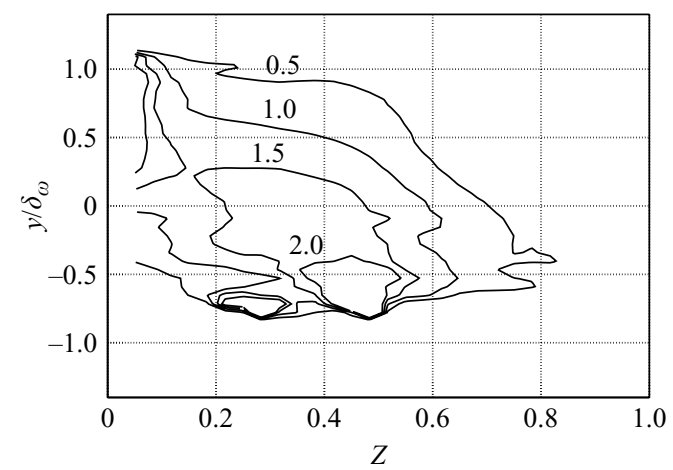

FIGURE 17. Scalar p.d.f. inside the upper turbulence interface at $\tau_{2}=183.0$. Contours of the function $P_{Z, i}(Z, \xi)$.
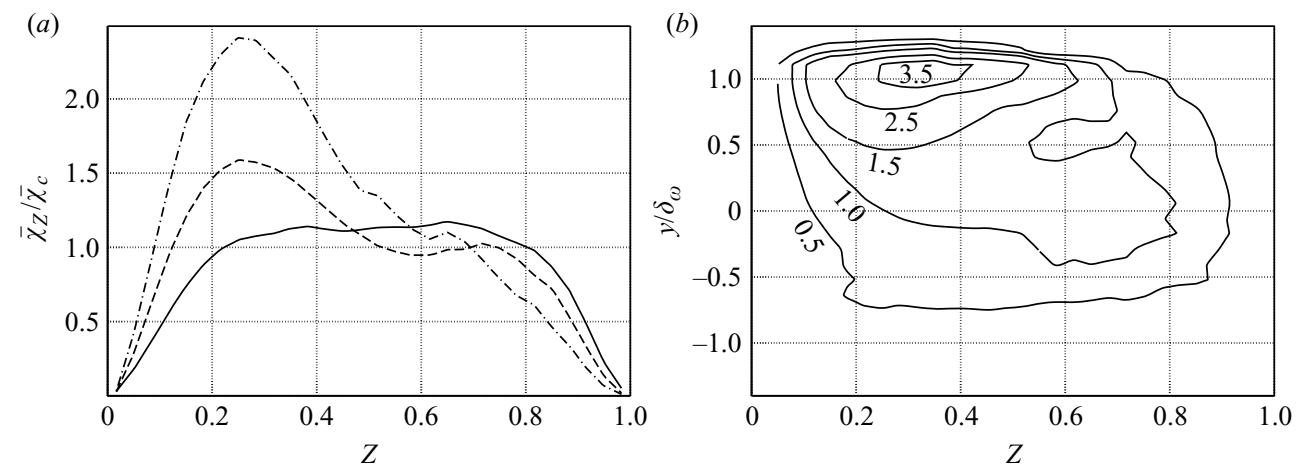

FIGURE 18. Conditional mean scalar dissipation rate inside the upper turbulence interface between $\tau_{1}$ and $\tau_{2}$. Profiles $(a)$ at different heights: solid, $y=0$; dashed, $y=\delta_{\omega} / 2$; dot-dashed, $y=3 \delta_{\omega} / 4$. Contours $(b)$ of the function $\bar{\chi}_{Z}(Z, \xi) / \bar{\chi}_{c}(t)$.

value. The detailed behaviour in the limit $Z \rightarrow 0$ depends on the threshold used to differentiate between the outer non-turbulent region and the upper interface, but it is not of importance for the conclusions drawn in the present study, as already mentioned in the previous section. As we move downward, the distribution shifts to intermediate values of the scalar and a maximum appears at about $Z=0.4$ which dominates the p.d.f. below the centre-plane. Note that the statistical convergence below $\xi=-0.5$ is very poor because the frequency of the upper interface being there is very small, as shown in the corresponding profile of zonal intermittency in figure 10 .

The variation in time of the scalar distribution is observed by comparing figure 16 at $\tau_{1}$ with figure 17 at $\tau_{2}$. As happened in the turbulent zone, there seems to be a trend towards a marching behaviour, meaning that a smoother variation with the lateral distance $y$ of the mean scalar emerges at later times, and the isocontours become more skewed. Higher Reynolds numbers are needed to confirm this trend.

The conditional mean scalar dissipation rate, conveniently scaled with $\bar{\chi}_{c}$, is presented in figure 18. The contour plot shows that this statistic increases first slowly between 0.5 and 1.5 in the central region of the mixing layer and develops then a strong peak between $Z=0.2$ and $Z=0.4$ as one approaches the upper free stream, beyond $\xi=0.5$. The profiles in figure $18(a)$ are similar to the total ones of figure 6 . The conditional standard deviation $\sigma_{\chi \mid Z}$ is shown in figure 19. The magnitude 
(a)

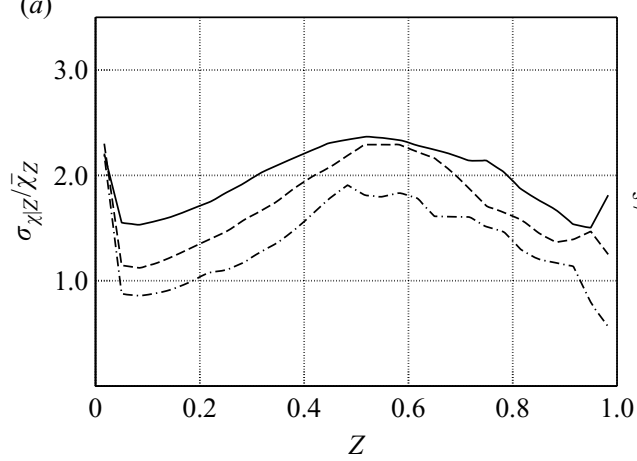

(b)

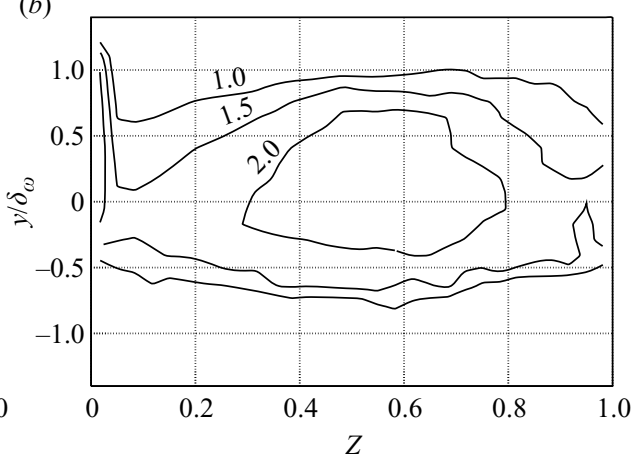

FIGURE 19. Ratio between conditional standard deviation $\sigma_{\chi \mid Z}$ and conditional mean inside the upper turbulence interface between $\tau_{1}$ and $\tau_{2}$. Profiles $(a)$ at different heights: solid, $y=0$; dashed, $y=\delta_{\omega} / 2$; dot-dashed, $y=3 \delta_{\omega} / 4$. Contours $(b)$ of the function $\left(\sigma_{\chi} \mid Z / \bar{\chi}_{Z}\right)(Z, \xi)$.

is again between 1.5 and 2.0 times larger than the conditional mean in the central region, for a height $y$ over the centre-plane between $-0.5 \delta_{\omega}(t)$ and $0.5 \delta_{\omega}(t)$. It is interesting to note that the maximum of $\sigma_{\chi \mid Z} / \bar{\chi}_{Z}$ occurs around the centre, and the standard deviation falls to zero where the conditional mean peaks. The temporal variation of these statistics, once represented in self-similar variables, does not show an appreciable change in the spatial structure as occurred with the p.d.f. (within the time interval considered), only a mild increase of the magnitude of the scalar dissipation rate around the centre-plane as in the turbulent zone (cf. figure 13). Thus, the corresponding statistics have been calculated using the average over the four times in order to improve the statistical convergence.

These observations are consistent with the general picture of outer fluid being brought towards the centre by large-scale motion (engulfing) and there being more strongly mixed with the turbulent flow (Bisset et al. 2002). This structure is also observed in the visualizations of figure 8 . If we consider first the passive scalar far from the centre $(\xi \simeq 1)$, it is characterized by a smooth variation between the outer value and the corresponding maximum, which leads to a concentration of the distribution near $Z=0$ as observed in figure 16 . This relatively smooth and fast transition of the scalar gives rise to a strong localized gradient and therefore to a high value of scalar dissipation rate, as observed in figure 18 for $\xi$ approaching 1 , without much fluctuation around that conditional mean and thus having a small $\sigma_{\chi \mid Z}$, as seen in figure 19. This behaviour could be interpreted in terms of a cliff structure existing at the top of the outward-moving bulges. On the other hand, when the upper interface is around the centre and mixes strongly, the scalar trajectory spends more time at intermediate values of the scalar (cf. figure 16), the gradient strongly fluctuating (cf. figure 19) and possibly having several maxima inside one trajectory (as reported by Peters \& Wang 2007), with a more distributed value of the dissipation rate (cf. figure 18). In addition to this engulfing process, figure 8 also shows that some smaller scale activity is occurring on top of these outward-moving bulges, and it can be argued that certain amount of nibbling is present (Mathew \& Basu 2002). This phenomenon would contribute to the intermediate values in the scalar p.d.f. and the conditional mean dissipation rate in the region $\xi=0.0-0.5$, in figures 16 and 18 . It seems reasonable to expect that both phenomena, engulfing and nibbling, appear, and the predominance of one or the other depends on different parameters like details 


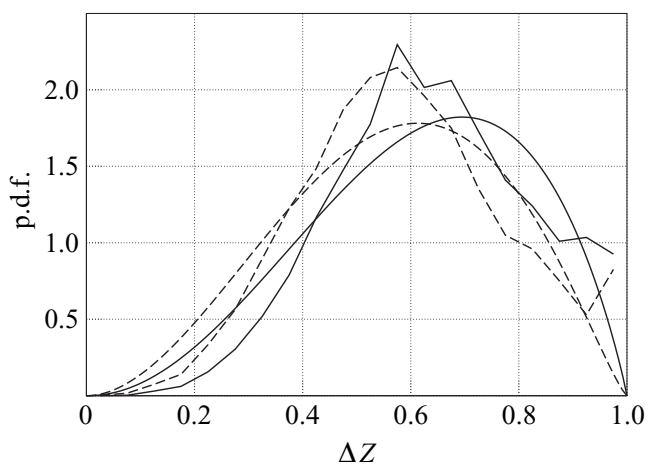

FiguRE 20. Distribution of the trajectory scalar difference inside the upper turbulence interface at different times: solid, $\tau_{1}=92.9$; dashed, $\tau_{2}=183.0$. Smooth lines are the corresponding beta p.d.f. approximations.

of the initial conditions, the ratio $\delta_{\omega}(t) / \delta_{\omega, 0}$ or the Reynolds number, which have a strong influence on the large-scale dynamics in free shear flows.

This behaviour of the conditional mean scalar dissipation rate and the corresponding standard deviation indicates that the relation between the local scalar dissipation rate, $\chi$, and the associated scalar field, $Z$, depends strongly on the lateral position also inside the turbulence interface, and therefore the joint p.d.f. between the scalar dissipation rate and the scalar varies significantly with the distance to the centre-plane, which might be of importance for turbulent combustion models. For instance, the conditional p.d.f. of the scalar dissipation $P_{\chi \mid Z}$ follows approximately a lognormal distribution (except for the tails) whose standard deviation has a one-toone relation with the ratio $\sigma_{\chi \mid Z} / \bar{\chi}_{Z}$; figure 19 shows a lateral variation of this quantity and therefore a lateral variation of the conditional distribution. This result indicates that the post-processing using the whole volume can lead to some inaccuracies, even for conditional variables, when external intermittency is important.

The last statistic computed inside the turbulence interface is the p.d.f. of the scalar difference along the trajectory. In principle this statistic could depend on the vertical distance $y$ to the centre-plane of the point associated to the trajectory, but the final extremum belongs to the boundary of the turbulent zone, which was observed to be approximately homogeneous in the time interval considered in this study, and therefore the same behaviour could be expected in this p.d.f. At the same time, statistical convergence of this distribution is poor because many different trajectories share the same extremum, and the sample size is smaller than in the other statistics considered in this work. Because of these two reasons the p.d.f. is constructed using the whole turbulence interface, the result being shown in figure 20. The beta p.d.f. approximation calculated with the first two moments of the mean profiles (mean and standard deviation equal to 0.62 and 0.20 , respectively, at time $\tau_{1}$ and equal to 0.57 and 0.20 at time $\tau_{2}$ ) is included. This information is important for flamelet models. The stoichiometric mixture fraction for commonly used hydrocarbon or hydrogen mixtures is a small number, less than 0.1 . Figure 20 tells us that the probability of the upper bound of the flamelet occurring at scalar values less than 0.1 is practically negligible, and then the stoichiometric surface lies practically always inside the turbulent/non-turbulent interface, although there is a certain shift of the distribution towards lower values of $Z$ as the time increases (i.e. Reynolds number increases), and longer simulations would be interesting. In addition, that same figure 

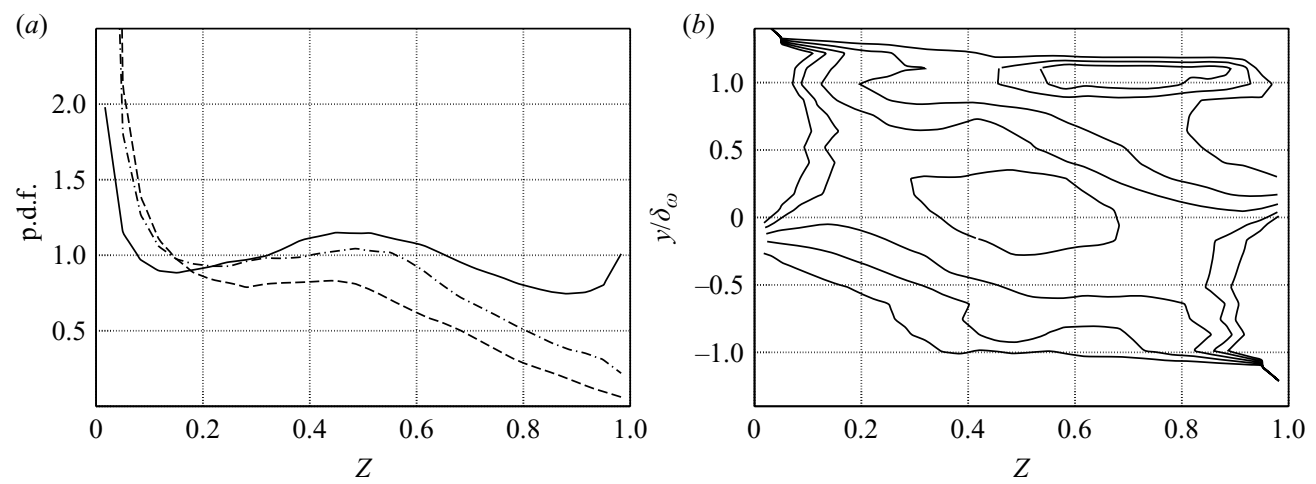

FIGURE 21. Scalar p.d.f. inside the quasi-laminar diffusion layers at $\tau_{1}=92.9$. Distributions (a) at different heights: solid, $y=0$; dashed, $y=\delta_{\omega} / 4$; dot-dashed, $y=\delta_{\omega} / 2$. Contours $(b)$ of the function $P_{Z}(Z, \xi)$ every 0.25 , starting at 0.25 .

gives the distribution of the upper bound of the flamelets and shows that the beta distribution deviates from the measured p.d.f.

\subsection{Quasi-laminar diffusion layers}

This last zone is defined by the points whose trajectories join the upper stream with the lower stream without ending in any intermediate extremal point and can be approximately identified with the braids between the billows observed in figure 8 . The statistics are presented at time $\tau_{1}$ and not at time $\tau_{2}$ because the sample size of this region decreases significantly for later times, as shown in figures 10 and 11; on the one hand statistical convergence deteriorates, and on the other hand the significance of this region for the whole shear layer decreases.

The corresponding scalar p.d.f. is shown in figure 21, presenting again the contour map and single profiles. The spatial distribution depicted by the contour plots is similar to that of the conventional statistic, shown in figure 4, having a maximum around the centre of the layer for $Z=0.5$ and a diagonal band joining the two delta functions that form at the limits $Z=0$ and $Z=1$. However, the central peak is here lower and the p.d.f. broader in scalar space, with a stronger banded structure. Results for later times suggest that this banded character becomes dominant inside this region.

The conditional mean scalar dissipation rate is presented in figure 22. Again, the spatial distribution is qualitatively the same as the corresponding conventional statistic, shown in figure 6 , having maxima at the edges of the mixing region, at about $y / \delta_{\omega}= \pm 1$, and an approximate central plateau. The difference is that the level of dissipation of this central plateau is higher here, about $1.5 \bar{\chi}_{c}-3.5 \bar{\chi}_{c}$, as well as the peaks at the upper and lower edges of the mixing region. Figure 23 shows that the variance of the conditional scalar dissipation across the mixing layer is more homogeneous than the conventional statistics of figure 7, with a magnitude about $50 \%$ larger than the conditional mean. These results are the motivation for the name of quasi-laminar diffusion layers. First, there are no maxima/minima of the scalar field inside them, as occurs in the turbulent zone, but they are not really laminar because of the strong fluctuation in the scalar dissipation. Second, the dissipation is large compared to the two other zones. The variation in time is again a tendency to homogenize the behaviour of these statistics in physical space, increasing the dissipation rate in the central region. 
(a)

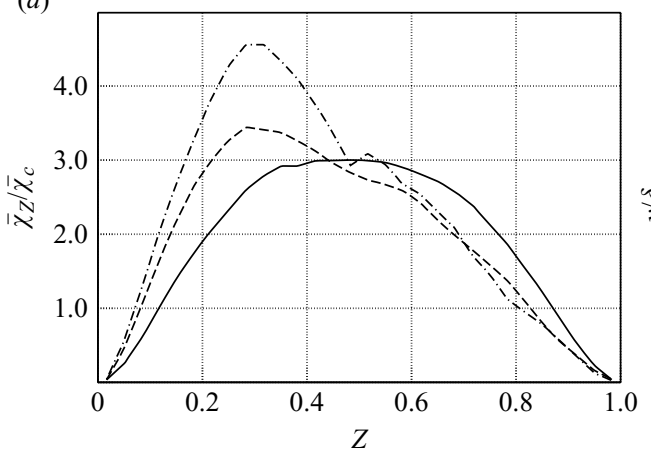

(b)

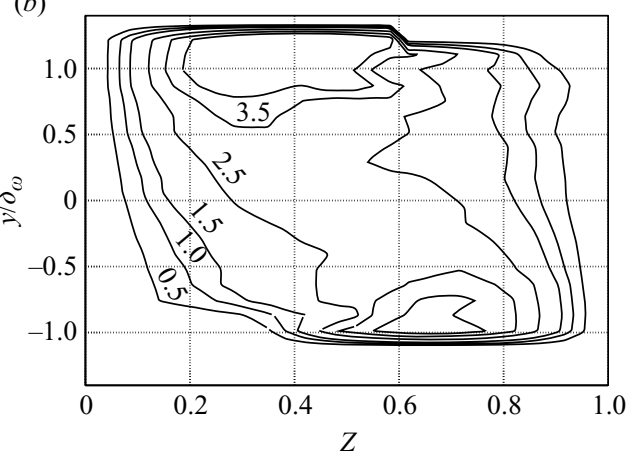

FIGURE 22. Conditional mean scalar dissipation rate inside the quasi-laminar diffusion layers at $\tau_{1}=92.9$. Profiles $(a)$ at different heights: solid, $y=0$; dashed, $y=\delta_{\omega} / 2$; dot-dashed, $y=3 \delta_{\omega} / 4$. Contours $(b)$ of the function $\bar{\chi}_{Z}(Z, \xi) / \bar{\chi}_{c}(t)$.
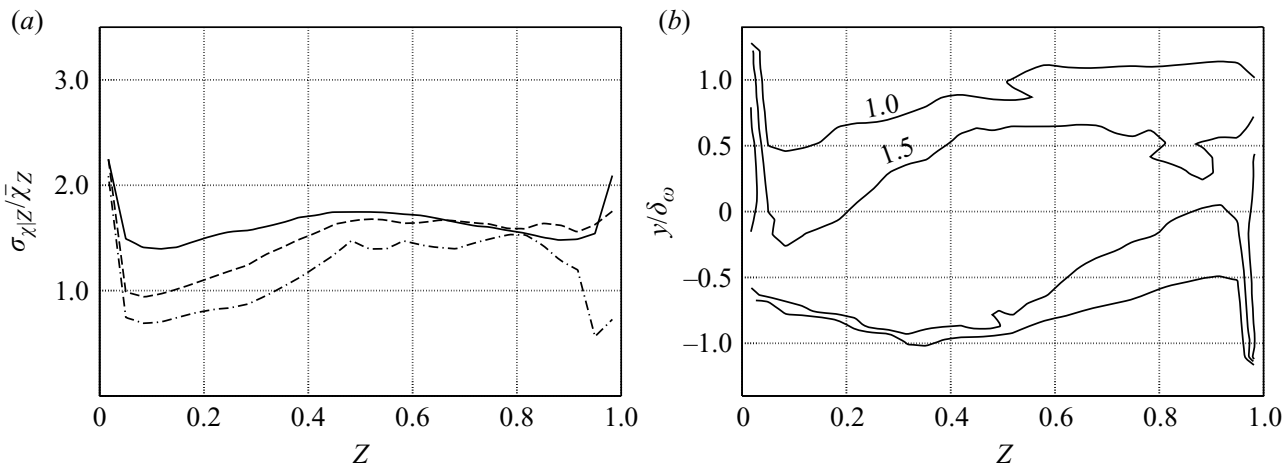

FiguRE 23. Ratio between conditional standard deviation $\sigma_{\chi \mid Z}$ and conditional mean inside the quasi-laminar diffusion layers at $\tau_{1}=92.9\left(\delta_{\omega} / \delta_{\omega, 0}=14.5\right)$. Profiles $(a)$ at different heights: solid, $y=0$; dashed, $y=\delta_{\omega} / 2$; dot-dashed, $y=3 \delta_{\omega} / 4$. Contours $(b)$ of the function $\left(\sigma_{\chi} \mid Z / \bar{\chi}_{Z}\right)(Z, \xi)$.

The variation of the conditional mean scalar dissipation rate is similar to that of the turbulence interfaces, with marked maxima at the edges $y= \pm \delta_{\omega}(t)$. However, there are differences as well. One difference is that the value of the dissipation rate inside these quasi-laminar diffusion layers is more than twice that of the turbulence interface for almost all the mixing layer extent, between $\xi=-0.9$ and $\xi=0.9$, and that the lateral peaks of scalar dissipation rate spread deeper along the $y$-coordinate. A second difference is that the conditional standard deviation is smaller in magnitude and more homogeneous in these quasi-laminar diffusion layers, which would simplify possible models in these regions. (A model of the form $\chi=\chi_{0} f(Z)$ might be more appropriate here.) This behaviour suggests the characterization of these braid zones in terms of strong cliff structures, which are then strained towards the top of the engulfing bulges to form part of the turbulence interface. This result is again consistent with the stagnation-point flow structure of these braid regions described in Bisset et al. (2002) and the visualizations presented in figure 8 .

\section{Discussion}

It has been shown that the mixing layer can be decomposed into four different zones, namely the outer non-turbulent regions, the turbulent zone, the turbulence interfaces 
and the quasi-laminar diffusion layers (cf. figure 10 in $\S 4$ ), a partition that will be used now to explain the conventional statistics, presented in $\S 3$, in terms of the zonal statistics, presented in $\S 5$. The difference with the conventional intermittency studies (Antonia 1981) is that the conventional turbulent region is here further separated into turbulence interfaces, turbulent zones and quasi-laminar diffusion layers, as originally proposed by Effelsberg \& Peters (1983). The separation among zones is defined by first maximum/minimum detection following gradient trajectories of the scalar field because of the significance for flamelet theory in turbulent combustion. This choice is supported a posteriori by the identification of a turbulent region reasonably well differentiated from the rest, with a homogeneous behaviour that allows to compare this zone for homogeneous turbulent flows, but other fields could be used in the future to track gradient trajectories. With respect to the turbulence interfaces and quasi-laminar layers, the distinction is not so clear, both sharing a similar qualitative spatial structure and the main difference being the level of scalar dissipation rate. However, the contribution of the quasi-laminar layers decreases as the Reynolds number increases, and the flow is represented by the first two zones, apart from the non-turbulent regions. The relative importance of each zone is provided by the zonal probabilities, or zonal intermittency factors, shown in figure 10. Although evolving in time, the turbulence interface represents most of the mixing layer, about $70 \%$, the turbulent zones about $25 \%$ and the quasi-laminar diffusion layers account for the remaining $5 \%$ at the final time considered in this work. The characteristics of the turbulence interfaces are, therefore, very important, specially for non-premixed turbulent combustion, since it has been shown (cf. figure 20 and the corresponding discussion) that the stoichiometric surface resides typically inside these regions. As already discussed in $\S 4$, this flow partition evolves in time as the Reynolds number increases, but no conclusion can be drawn since the range of Reynolds numbers achieved in the present simulation is moderate, $R e_{\omega} \simeq 3000$.

With respect to the scalar p.d.f., the banded structure of the function $P(Z, \xi)$ shown in figure 4 is mainly due to the distribution inside the upper turbulence interface, shown in figure 16 , which contributes most between $\xi=-0.5$ and $\xi=1$ according to figure 10, and the corresponding part from the lower interface. This banded structure is reinforced by the quasi-laminar diffusion layers (figure 21). Finally, the turbulent regions add to the central peak, according to the distribution shown in figure 12 . The marching behaviour seems to become prevalent for later times inside all the identified zones, but longer simulations are needed to reach a conclusion. Of course, as one moves into the outer stream, the approximate delta function at the corresponding free-stream value dominates the scalar p.d.f. Note in figure 11 that the reduction with time of the volume occupied by the quasi-laminar diffusion layer is compensated at early times and to a large extent by the increase in the volume occupied by the turbulence interfaces, which, both having similar spatial structure, implies only a small variation in time in the conventional statistic shown in figure 5.

Similar reasoning is done for the conditional mean scalar dissipation rate. The central plateau of the function $\bar{\chi}_{Z}(Z, \xi)$ in figure 6 , with values between $0.5 \bar{\chi}_{c}(t)$ and $1.5 \bar{\chi}_{c}(t)$, corresponds mainly to the dissipation rate inside the turbulence interfaces, figure 18. The peaks of dissipation rate around the edge of the mixing layer, at a distance $\delta_{\omega}(t)$ from the centre-plane and with a magnitude larger than $3.5 \bar{\chi}_{c}(t)$, originate also in this interface. This general spatial distribution is reinforced again by the quasi-laminar diffusion layers, as observed in figure 22. The contribution from the turbulent region is less important, not only because the relative volume occupied by the zone is small but also because the level of dissipation there is significantly smaller, 
of the order of $0.4-0.6 \bar{\chi}_{c}(t)$, as seen in figure 13. The visualizations of figure 8 also show that the stronger dissipation events occur in the interface and braids of the shear layer. The lateral variation of the conditional mean dissipation rate, when integrated across the shear layer, leads to the double hump observed in the volume-averaged profiles shown in figure $3(b)$.

Hence, it seems that the presence of external intermittency is the main cause for the crosswise variation of the statistics considered in this paper, since this variation resides mainly in the turbulence interfaces, and not only a secondary quantity as suggested in the past (Anselmet et al. 1994; Mi \& Antonia 1995). For instance, variation of the skewness of the scalar p.d.f. or the conditional mean scalar dissipation rate are both manifestations of the same phenomenon, external intermittency. When external intermittency is not present, this spatial variation seems to be strongly reduced, in spite of having an inhomogeneity in some direction (Bilger 1993; Mell et al. 1994; Markides \& Mastorakos 2006).

The turbulent zone, though contributing little to the conventional statistics studied here, shows a very notorious behaviour, being approximately homogeneous but not isotropic. The scalar distribution shows exponential tails, which is linked to the $V$-shaped profile of $\bar{\chi}_{Z}(Z)$ and agrees with data in the intermediate state of a decaying initially bimodal scalar distribution in the stationary isotropic turbulence DNS by Eswaran \& Pope (1988), with the difference that, here, the scalar difference is continuously fed through turbulent entrainment.

Implications for turbulent combustion modelling have been also indicated through the paper: $(a)$ conditioning of $\chi$ on $Z$ does not reduce the fluctuation levels of the former; $(b) \chi$ and $Z$ are not independent, as indicated by some results in the past, but their relation is not satisfactorily described by $\chi=\chi_{0} f(Z)$, with $\chi_{0}$ and $Z$ independent and $f$ any deterministic function; $(c)$ volume analysis, e.g. to calculate $P_{\chi \mid Z}\left(\chi \mid Z=Z_{s t}\right)$ or $\bar{\chi}_{Z}$ at the stoichiometric surface, could miss some of this important spatial variation. With respect to this last point, it is observed that $\bar{\chi}_{Z}$ is underpredicted by a factor of 2 or 3 when the complete field is used. However, it needs to be remarked that the density in this work is approximately constant, and it is known that the general structure of the flow changes when heat release effects are retained; Pantano et al. (2003) show how the local modification of the density fields through heat release reduces the external intermittency of the shear layer, since the turbulent flow has less inertia compared to the outer non-turbulent flow. This phenomenon would prevent the turbulence interfaces to penetrate as deep into the mixing region as reported here, leading to a smaller variation across the free shear flow, as reported by measurements (Chen \& Mansour 1997; Starner et al. 1997), though there would be some distance from the centre-plane at which the turbulence interface sits, and then stronger variations of conditional moments of $\chi$ should be observed. Nevertheless, the present analysis of a cold flow is a required first step, since detailed report of these quantities using DNS had not been done yet. Analysis of DNS of reacting flows, which are already becoming available (Pantano 2004; Hawkes et al. 2007), would be of interest.

\section{Conclusions}

Gradient trajectory analysis has been applied to the passive scalar field $Z$ of a temporally evolving shear layer in order to study the scalar distribution $P_{Z}(Z ; y, t)$, the conditional mean scalar dissipation rate $\bar{\chi}_{Z}(Z ; y, t)$ and the corresponding standard deviation $\sigma_{\chi \mid Z}(Z ; y, t)$ in the presence of external intermittency. The Schmidt number is 1 . The resolution requirements needed are $\eta / \Delta x$ greater than 
unity, where $\eta$ is the Kolmogorov scale and $\Delta x$ the grid spacing, for a numerical algorithm using sixth-order compact schemes for the spatial derivatives and fourthorder Runge-Kutta in time.

Results of this non-local analysis have shown that the conventional turbulent region of free shear flows can be further partitioned into a turbulent zone, defined by points whose trajectory connects one minimum with one maximum, and a turbulence interface (or interfaces), defined by points with trajectories moving from the outer non-turbulent streams towards a first extremum. The turbulent zones so identified show a distinct behaviour, homogeneous but anisotropic, with a mean scalar gradient almost zero for the major part of the time interval considered in this work. In addition, quasi-laminar diffusion layers, with points whose trajectory moves directly from one free stream to the other, are observed in the early times of the development. It is seen that the turbulence interfaces often cross the centre of the mixing region until distances $0.5 \delta_{\omega}$ below and above the centre-plane, respectively. This partition splits the conventional intermittency factor in several contributions. For the Reynolds numbers considered in this work, of the order of 3000 based on the vorticity thickness, the volume fraction occupied by the turbulence interfaces is the greatest, about three times that of the turbulent zones. Hence, the characteristics of the fields inside the turbulence interfaces determine to a large extent the conventional statistics of the flow. As the Reynolds number increases, so does the volume occupied by the turbulent zones but at a small rate.

The spatial structure of the scalar p.d.f. and of the first two moments of the conditional scalar dissipation rate is well characterized by the instantaneous vorticity thickness $\delta_{\omega}(t)$ and the mean dissipation rate at the centre-plane $\bar{\chi}_{c}(t)$. The scalar p.d.f. shows the usual hybrid structure, between pure marching and non-marching types, although the marching behaviour strengthens at later times in all the zonal distributions. The conditional mean scalar dissipation rate $\bar{\chi}_{Z}$ also depends strongly on $y$. In particular, it shows a central plateau, which is the main contribution to the unconditional mean dissipation, and two strong peaks at the edges of the mixing layer, at a distance about $\delta_{\omega}(t)$ and with a magnitude about three times that of the centre-plane value. On the other hand, the conditional standard deviation is relatively larger in the central region and smaller where the conditional mean peaks. This spatial behaviour is mainly due to the turbulence interfaces and the quasi-laminar diffusion layers, and the turbulent zones contribute only moderately. It has been argued that the strong peaks are due to the formation of cliff structures in the quasi-laminar diffusion layers and the external region of the turbulence interface.

Another result of significance for the flamelet model in non-premixed turbulent combustion is that the scalar difference along trajectories starting in the outer stream is almost always greater than 0.1 at this Reynolds number. That implies that the surface of stoichiometric mixture fraction lies inside the turbulence interface for many hydrocarbon-air and hydrogen-air mixtures, and it is the scalar dissipation inside these regions that needs to be accurately modelled; the turbulent zone is less important, and therefore, the application of results derived only from homogeneous turbulence is limited.

We thank Professor A. Tsinober and Professor S. Sarkar for useful and motivating discussions on the topic. The contribution from J. H. Göbbert by providing part of the parallel post-processing routines is gratefully acknowledged. Financial support for this work was provided by the Deutsche Forschungsgemeinschaft under grant Pe 241/30-2 and computational time by the Jülich Supercomputer Center. 


\section{REFERENCES}

Anselmet, F., Djeridi, H. \& Furlachier, L. 1994 Joint statistics of a passive scalar and its dissipation in turbulent flows. J. Fluid Mech. 280, 173-197.

Antonia, R. A. 1981 Conditional sampling in turbulence measurements. Annu. Rev. Fluid Mech. 13, 131-156.

Bilger, R. W. 1993 Conditional moment closure for turbulent reacting flow. Phys. Fluids 5 (2), 436-444.

Bilger, R. W. 2000 Future progress in turbulent combustion research. Prog. Energy Combust. Sci. 26, 367-380.

Bisset, D. K., Hunt, J. C. R. \& Rogers, M. M. 2002 The turbulent/non-turbulent interface bounding a far wake. J. Fluid Mech. 451, 383-410.

Carpenter, M. H., Gottlieb, D. \& Abarbanel, S. 1993 The stability of numerical boundary treatments for compact high-order finite-difference schemes. J. Comput. Phys. 108, 272-295.

Chen, Y. C. \& Mansour, M. S. 1997 Measurements of scalar dissipation in turbulent hydrogen diffusion flames and some implications on combustion modelling. Combust. Sci. Technol. 126, 291-313.

Cook, A. W., Riley, J. J. \& Kosály, G. 1997 A laminar flamelet approach to subgrid-scale chemistry in turbulent flows. Combust. Flame 109, 332-341.

Corrsin, S. \& Kistler, A. L. 1955 Free-stream boundaries of turbulent flows. Tech Rep. 1244. NACA.

Cortesi, A. B., Smith, B. L., Sigg, B. \& Banerjee, S. 2001 Numerical investigation of the scalar probability density function distribution in neutral and stably stratified mixing layers. Phys. Fluids 13 (4), 927-950.

Dimotakis, P. E. 2000 The mixing transition in turbulent flows. J. Fluid Mech. 409, 69-98.

Dimotakis, P. E. 2005 Turbulent mixing. Annu. Rev. Fluid Mech. 37, 329-356.

Effelsberg, E. \& Peters, N. 1983 A composite model for the conserved scalar PDF. Combust. Flame 50, 351-360.

EfFelsberg, E. \& Peters, N. 1988 Scalar dissipation rates in turbulent jets and jet diffusion flames. In Twenty-Second Symp. (Intl) on Combustion. Seatle, USA.

Erlebacher, G., Hussaini, M. Y., Kreiss, H. O. \& Sarkar, S. 1990 The analysis and simulation of compressible turbulence. Theor. Comput. Fluid Dyn. 2, 73-95.

Eswaran, V. \& Pope, S. B. 1988 Direct numerical simulations of the turbulent mixing of a passive scalar. Phys. Fluids 31 (3), 506-520.

Hawkes, E. R., Sankaran, R., Sutherland, J. C. \& Chen, J. H. 2007 Scalar mixing in direct numerical simulations of temporally evolving plane jet flames with skeletal $\mathrm{CO} / \mathrm{H}_{2}$ kinetics. Proc. Combust. Inst. 31, 1633-1640.

Holzner, M., Liberzon, A., Guala, M., Tsinober, A. \& Kinzelbach, W. 2006 Generalized detection of a turbulent front generated by an oscillating grid. Exp. Fluids 41, 711-719.

Holzner, M., Liberzon, A., Nikitin, N., Kinzelbach, W., LÜthi, B. \& Tsinober, A. 2008 A Langrangian investigation of the small-scale features of turbulent entrainment through particle tracking and direct numerical simulation. J. Fluid Mech. 598, 465-475.

Holzner, M., Liberzon, A., Nikitin, N., Kinzelbach, W. \& Tsinober, A. 2007 Small-scale aspects of flows in proximity of the turbulent/nonturbulent interface. Phys. Fluids 19, 071702.

Hunt, J. C. R., Eames, I. \& Westerweel, J. 2006 Mechanics of inhomogeneous turbulence and interfacial layers. J. Fluid Mech. 554, 499-519.

JAYESH \& WARHAFT, Z. 1992 Probability distribution, conditional dissipation and transport of passive temperature fluctuations in grid-generated turbulence. Phys. Fluids A 4 (10), 2292-2307.

Kovasznay, L. S. G., Kibens, V. \& Blackwelder, R. F. 1970 Large-scale motion in the intermittent region of a turbulent boundary layer. J. Fluid Mech. 41, 283-325.

Kravchenko, A. G. \& Moin, P. 1997 On the effect of numerical errors in large-eddy simulations of turbulent flows. J. Comput. Phys. 131, 310-322.

Kushnir, D., Schumacher, J. \& Brandt, A. 2006 Geometry of intensive scalar dissipation events in turbulence. Phys. Rev. Lett. 97 (124502).

LaRue, J. C. \& Libby, P. A. 1976 Statistical properties of the interface in the turbulent wake of a heated cylinder. Phys. Fluids 19 (12), 1864-1875. 
LeLE, S. K. 1992 Compact finite difference schemes with spectral-like resolution. J. Comput. Phys. 103, 16-42.

Libby, P. A. \& Williams, F. A., ed. 1994 Turbulent Reacting Flows. Academic.

Markides, C. N. \& Mastorakos, E. 2006 Measurements of the statistical distribution of the scalar dissipation rate in turbulent axisymmetric plumes. In Turbulence, Heat and Mass Transfer (ed. Y. Nagano, K. Hanjalić \& S. Jakirlić), vol. 5, pp. 1-20. Begall House Inc.

Mathew, J. \& Basu, A. J. 2002 Some characteristics of entrainment at a cylindrical turbulence boundary. Phys. Fluids 14 (7), 2065-2072.

Mell, W. E., Nilsen, V., Kosály, G. \& Riley, J. J. 1994 Investigation of closure models for nonpremixed turbulent reacting flows. Phys. Fluids 6 (3), 1331-1356.

Mellado, J. P., Friedrich, R. \& Sarkar, S. 2007 Modelling of the filtered heat release for large eddy simulation of compressible infinitely fast reacting flows. Proc. Combust. Inst. 31, 16911699.

Mi, J. 2006 Correlation between non-Gaussian statistics of a scalar and its dissipation rate in turbulent flows. Phys. Rev. E 74 (016301).

Mi, J. \& Antonia, R. A. 1995 Joint statistics between temperature and its dissipation rate components in a round jet. Phys. Fluids 7 (7), 1665-1673.

O'Brien, E. E. \& JiAng, T. 1991 The conditional dissipation rate of an initially binary scalar in homogeneous turbulence. Phys. Fluids A 3 (12), 3121-3123.

Pantano, C. 2004 Direct simulation of nonpremixed flame extinction in a methane-air jet with reduced chemistry. J. Fluid Mech. 514, 231-270.

Pantano, C. \& SaRkar, S. 2002 A study of compressibility effects in the high-speed, turbulent shear layer using direct simulation. J. Fluid Mech. 451, 329-371.

Pantano, C., Sarkar, S. \& Williams, F. A. 2003 Mixing of a conserved scalar in a turbulent reacting shear layer. J. Fluid Mech. 481, 291-328.

Peters, N. 2000 Turbulent Combustion. Cambridge University Press.

Peters, N. \& Trouillet, P. 2002 On the role of quasi-one-dimensional dissipation layers in turbulent scalar mixing. In Annual Research Briefs, pp. 27-40. Center for Turbulence Research. Stanford University.

Peters, N. \& WANG, L. 2007 The central role of scalar dissipation rate in nonpremixed combustion. In Proc. Fifth US Combustion Meeting. San Diego, USA.

Picket, L. M. \& Ghandhi, J. B. 2002 Passive scalar mixing in a planar shear layer with laminar and turbulent inlet conditions. Phys. Fluids 14 (3), 985-998.

Pitsch, H., Chen, M. \& Peters, N. 1998 Unsteady flamelet modeling of turbulent hydrogen-air difussion flames. Twenty-Seventh Symp. (Intl) on Combustion. Boulder, USA.

Rogers, M. M. \& Moser, R. D. 1994 Direct simulation of a self-similar turbulent mixing layer. Phys. Fluids A 6 (2), 903-923.

Sreenivasan, K. R. \& Antonia, R. A. 1997 The phenomenology of small-scale turbulence. Annu. Rev. Fluid Mech. 29, 435-472.

Sreenivasan, K. R., Ramshankar, R. \& Meneveau, C. 1989 Mixing, entrainment and fractal dimensions of surfaces in turbulent flows. Proc. R. Soc. Lond. A 421, 79-108.

Stanley, S. A., Sarkar, S. \& Mellado, J. P. 2002 A study of the flow-field evolution and mixing in a planar turbulent jet using direct numerical simulation. J. Fluid Mech. 450, 377 407.

Starner, S. H., Bilger, R. W., Long, M. B., Frank, J. H. \& Marran, D. F. 1997 Scalar dissipation measurements in turbulent jet diffusion flames of air diluted methane and hydrogen. Combust. Sci. Technol. 129, 141-163.

Su, L. K. \& Clemens, N. T. 2003 The structure of fine-scale mixing in gas-phase planar turbulent jets. J. Fluid Mech. 488, 1-29.

Thompson, K. W. 1990 Time-dependent boundary conditions for hyperbolic systems II. J. Comput. Phys. 89, 439-461.

Tong, C. \& Warhaft, Z. 1995 Passive scalar dispersion and mixing in a turbulent jet. J. Fluid Mech. 292, $1-38$.

Townsend, A. A. 1948 Local isotropy in the turbulent wake of a cylinder. Aust. J. Sci. Res. A 1 (2), $161-174$.

Townsend, A. A. 1976 The Structure of Turbulent Shear Flow. Cambridge University Press. 
Vedula, P., Yeung, P. K. \& Fox, R. O. 2001 Dynamics of scalar dissipation in isotropic turbulence: a numerical and modeling study. J. Fluid Mech. 433, 29-60.

Wang, L., Chen, S. \& Brasseur, J. G. 1999 Examination of hypothesis in the Kolmogorov refined turbulence theory through high-resolution simulations. Part 2. Passive scalar field. J. Fluid Mech. 400, 163.

WANG, L. \& Peters, N. 2006 The length-scale distribution function of the distance between extremal points in passive scalar turbulence. J. Fluid Mech. 554, 457-475.

Warhaft, Z. 2000 Passive scalars in turbulent flows. Annu. Rev. Fluid Mech. 32, 203-240.

Westerweel, J., Fukushima, C., Perdersen, J. M. \& Hunt, J. S. R. 2005 Mechanics of the turbulent-nonturbulent interface of a jet. Phys. Rev. Lett. 95 (174501), 1-4.

Westerweel, J., Hofmann, T., Fukushima, C. \& Hunt, J. C. R. 2002 The turbulent/non-turbulent interface at the outer boundary of a self-similar jet. Exp. Fluids 33, 873-878.

Williamson, J. H. 1980 Low-storage Runge-Kutta schemes. J. Comput. Phys. 35, 48-56. 